\title{
Crystallization in three- and two-dimensional colloidal suspensions
}

\author{
U Gasser \\ Laboratory for Neutron Scattering, ETH Zurich and Paul Scherrer Institut, \\ CH-5232 Villigen PSI, Switzerland \\ and \\ Adolphe Merkle Institut, University of Fribourg, PO Box 209, CH-1723 Marly 1, Switzerland \\ E-mail: urs.gasser@psi.ch
}

\begin{abstract}
Despite progress in the understanding of crystal nucleation and crystal growth since the first theories for nucleation were developed, an exact quantitative prediction of the nucleation rates in most systems has remained an unsolved problem. Colloidal suspensions show a phase behavior that is analogous to atomic or molecular systems and serve accordingly as ideal model systems for studying crystal nucleation with an accuracy and depth on a microscopic scale that is hard to reach for atomic or molecular systems. Due to the mesoscopic size of colloidal particles they can be studied in detail on the single-particle level and their dynamics is strongly slowed down in comparison with atomic or molecular systems, such that the formation of a crystal nucleus can be followed in detail. In this review, recent progress in the study of homogeneous and heterogeneous crystal nucleation in colloids and the controlled growth of crystalline colloidal structures is reviewed. All this work has resulted in unprecedented insights into the early stage of nucleation and it is also relevant for a deeper understanding of soft matter materials in general as well as for possible applications based on colloidal suspensions.
\end{abstract}

\section{Contents}

1. Introduction and overview

1.1. Fluid-solid transition

1.2. Criteria for freezing and melting

1.3. Freezing and melting in two dimensions

1.4. Interactions and comparison with atomic systems

2. Hard spheres

2.1. Homogeneous nucleation of HS crystal

2.2. Critical nuclei

2.3. Polydispersity

3. Freezing and melting in two dimensions

3.1. Melting of two-dimensional crystals according to KTHNY theory

3.2. Crystallization in two dimensions

4. Crystal nucleation of short, rod-shaped particles
5. Soft repulsive and attractive interactions 12

5.1. Crystal-crystal coexistence 12

5.2. Soft particles 13

6. Binary crystals $\quad 14$

6.1. Binary hard sphere crystals 14

6.2. Binary crystals of charged particles 15

7. Crystallization on substrates and in external fields 16 7.1. Substrates 17

7.2. External fields 19

8. New directions 20

Acknowledgments $\quad 20$

References $\quad 20$

\section{Introduction and overview}

10 As for other phase transitions, crystallization is a central 12 topic of condensed matter physics. It is at the heart of 
a thorough understanding of the controlled fabrication of crystalline materials and, therefore, is of great importance for many applications. In particular, crystals consisting of colloidal particles $[1,2]$ are of interest for developing novel materials such as photonic bandgap materials [3,4] or optical filters [5] that form by self-assembly. Furthermore, colloidal materials offer unique possibilities for studying the nucleation and growth of crystals on a microscopic scale, since the 'atoms' of the system are of colloidal size and, therefore, are easier to observe than atoms or molecules [6, 7].

The focus of this review lies in recent advances in the understanding of the fluid-crystal nucleation and growth process and on methods for controlling the structure and orientation of crystals. This review is organized as follows: in the remainder of this introduction, an overview of the fluid-crystal transition is given, criteria for freezing and melting are discussed, the special properties of crystals and phase transitions in two dimensions (2d) are introduced, and a comparison of colloidal systems with atomic systems, i.e. systems with building blocks of atomic or molecular size, is given. A review of the nucleation and growth of crystals in hard sphere (HS) systems is given in section 2. Recent findings about the properties of subcritical and critical crystal nuclei, the effect of polydispersity on nucleation and crystal growth are discussed. Insights into melting and freezing in $2 \mathrm{~d}$ systems are presented in section 3. Difficulties in the understanding of crystal nucleation in hard rods are presented in section 4 . In section 5, differences between systems with HS and soft interactions are presented and the possibility of crystal-crystal coexistence in systems with particular attractive interactions is reviewed. The formation of binary crystals is discussed in section 6 for HS systems and charged colloidal particles. An overview of the effects of external potentials due to substrates or external fields on nucleation and crystal growth in $2 \mathrm{~d}$ and $3 \mathrm{~d}$ is given in section 7 .

\subsection{Fluid-solid transition}

Generally, the formation of crystals in a metastable bulk liquid involves the creation of crystal nuclei containing a relatively large number of particles; the size of such nuclei is of the order of 10 particle diameters or larger. Such large initial nuclei are necessary, because the structures of the liquid and the crystal are fundamentally different and involve the formation of an energetically unfavorable interface between crystal and liquid. This interface gives rise to a free energy penalty $A \gamma$, which is proportional to the area $A$ of the interface and the surface energy $\gamma$. As a consequence, there is a free energy difference of crystal and fluid that has the form of a barrier and controls the nucleation process:

$$
\Delta G=G_{\text {crystal }}-G_{\text {fluid }}=A \gamma-V n_{\mathrm{s}} \Delta \mu,
$$

where $V$ is the volume of the crystal nucleus, $n_{\mathrm{s}}$ is the number density of particles in the crystal and $\Delta \mu=\mu_{\text {fluid }}-$ $\mu_{\text {crystal }}$ is the difference of the chemical potentials of fluid and crystal. This free energy barrier is sketched in figure 1; it is only overcome when a nucleus reaches the critical size, beyond which the favorable lower chemical potential of the

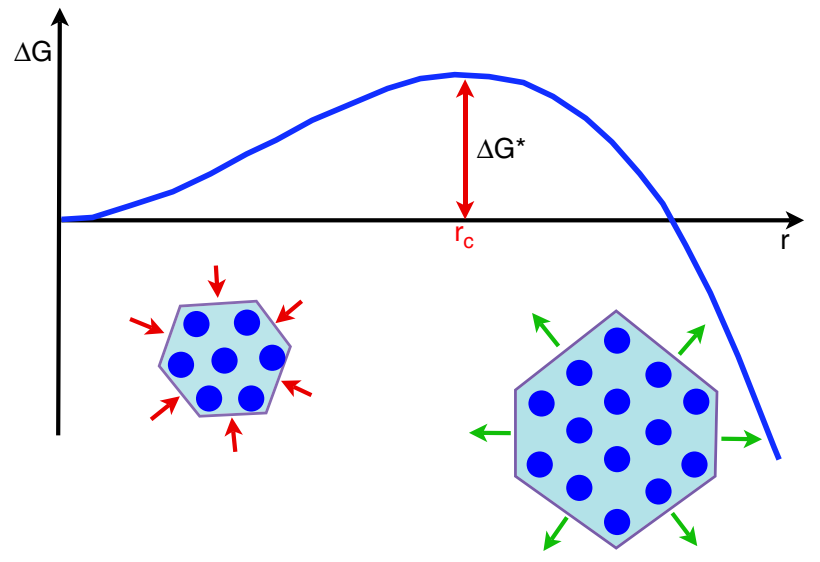

Figure 1. Free energy barrier of crystal nuclei. On average, nuclei of subcritical size $\left(r<r_{\mathrm{c}}\right)$ shrink, while nuclei that reach a postcritical size $\left(r>r_{\mathrm{c}}\right)$ can decrease their energy by growing.

crystal dominates and the energy is reduced when the crystal grows. The spontaneous formation of a critical nucleus in the bulk of an undercooled fluid-homogeneous nucleationis generally a rare event, since it happens due to random structural fluctuations. The formation of a nucleus on a seedheterogeneous nucleation-can be much faster, if the seed gives rise to a reduction of the free energy barrier.

The free energy barrier is the basis of nucleation theory. Classical nucleation theory (CNT), which assumes that nucleation and growth can be described using the properties of bulk crystal, was developed by Volmer and Weber [8] as well as Becker and Döring [9] for condensation in vapors and was extended to nucleation of crystals in liquids by Turnbull and Fisher [10]. The assumptions of CNT about small crystal nuclei are not based on a firm experimental foundation. The large differences between theoretical and experimental results for the maximum undercooling and nucleation rates in metal melts [11] are believed to be connected to the crude approximations for small crystals with a size of the order of the critical nucleus. Furthermore, it is not clear that the structure of the bulk crystal is the one with the smallest free energy barrier. It was already remarked by Ostwald [12] that the structure that forms initially need not be the one of the bulk crystal but is the one that forms fastest in the vicinity of the crystal-liquid interface, or on a seed in the case of heterogeneous nucleation. Recent findings about the properties of small crystal nuclei are discussed in subsection 2.2.

Before homogeneous crystal nucleation starts, crystal precursors and subcritical crystal nuclei form during an induction time. The length of this period depends on the ability of the system to stay in a metastable state. When nuclei cross the free energy barrier, these nuclei begin to grow continuously, while new critical nuclei continue to form. Steady state growth can be observed, during which the nucleation density rate keeps constant, while the postcritical nuclei grow [13]. Crystal growth, discussed in section 2.1, is expected to continue until crystals fill the whole volume of the sample and begin to interact. During the following ripening or coarsening of the crystals, large crystallites grow at the expense of smaller ones. The beginning of ripening is often observed before steady state 
growth of the crystals is reached [14, 15]. This regime is beyond the scope of this review.

\subsection{Criteria for freezing and melting}

Since free energy calculations are often not available, empirical rules for crystallization or melting that are experimentally easily accessible have been identified. The oldest is the Lindemann criterion for melting [16], which states that the root-mean-square deviation of a particle, which is given by the Lindemann parameter $L=\sqrt{\left\langle\Delta r^{2}\right\rangle} / a$, reaches a value of 0.15 when the crystal melts. Here, $\Delta r$ represents the displacement of a particle from its lattice position and $a$ is the average distance between nearest neighbors. This rule is not valid for crystals in two dimensions (2d), which have quasi-longrange instead of long-range translational order [17]. However, an adapted form of the Lindemann parameter can be used in 2d [18]. Remarkably, a theoretical study of hard spheres (HS) in spaces with more than three dimensions indicates [19] that the Lindemann criterion for melting is valid for dimensions up to at least 50. The Hansen-Verlet criterion [20] can be applied in both $2 \mathrm{~d}$ and $3 \mathrm{~d}$. At freezing the first peak of the structure factor $S(k)$ of a monatomic fluid reaches a height of 2.85 in $3 \mathrm{~d}$ and $\approx 4$ in $2 \mathrm{~d}$. A criterion that appears to be more general in the sense that it applies without restriction both in $2 \mathrm{~d}$ and $3 \mathrm{~d}$ is the Löwen-Palberg-Simon criterion [21]; the ratio of the long-time and the short-time diffusion constants, $D_{\mathrm{L}} / D_{\mathrm{S}}$, in the liquid is $\approx 0.1$ at freezing.

The beginning of crystal nucleation in a fluid can be detected by determining the pair distribution function $g(r)$ either with scattering or imaging techniques. A shoulder on the low- $r$ side of the second peak of $g(r)$ of a fluid is observed in HS in $2 \mathrm{~d}$ and $3 \mathrm{~d}$, and this shoulder has been shown to be related to the rearrangement of next-nearest neighbors when structural precursors of the crystal form [22]. In $2 \mathrm{~d}$, the shoulder corresponds clearly to the second peak position of $g(r)$ of the crystal. However, for $3 \mathrm{~d}$ fcc crystals the position of the shoulder corresponds to the third crystal peak in $g(r)$. This shoulder in $g(r)$ is an important test for theories describing the transition from fluid to solid. Many approximations used for the fluid state are not reliable close to the transition to the solid, e.g. with the Percus-Yevick and the mean spherical approximation for $g(r)$ the shoulder is not obtained. Close to the freezing transition triplet correlations have to be taken into account next to pair correlations [23]. Next to HS, the same shoulder in $g(r)$ is also observed for the $2 \mathrm{~d}$ Lennard-Jones fluid just before crystallization. However, it is not clear, whether it is a truly general feature that is observed for all kinds of interactions.

Due to their good experimental accessibility colloids are ideal for studying crystallization in two dimensions (2d). Important properties of $2 \mathrm{~d}$ crystals are presented in section 1.3 and studies of melting and freezing in $2 \mathrm{~d}$ are discussed in section 3. As explained below, crystals do not exist in onedimensional (1d) systems due to fluctuations. Freezing in more than three dimensions has been studied theoretically for HS [19]. For all dimensions $d>3$ a first-order transition is found that preempts a second-order (spinodal decomposition) instability that was previously reported [24].

\subsection{Freezing and melting in two dimensions}

In general, the phase behavior in two-dimensional (2d) and three-dimensional (3d) systems is expected to be similar in the sense that the crystal should form by a nucleation process. However, the situation is more complicated, since no true longrange translational order exists in $2 \mathrm{~d}$ crystals and the phase behavior close to freezing has been found to be richer than in 3d systems (see also section 3 ).

That no long-range translational order can exist in $2 \mathrm{~d}$ crystals was first shown by Peierls for the magnetic $X Y$ model [25]. Fluctuations with long wavelength destroy true long-range order. For a spin wave in a ferromagnetic system this can be understood quite easily. The energy it costs to tilt a spin by a small angle $\Delta \phi$ relative to its neighbors is proportional to $\Delta \phi^{2}$. With the nearest-neighbor distance $d$ and the wavelength $L=N d$ of the spin wave, the energy of this long wavelength fluctuation is proportional to $N^{2}(2 \pi / N)^{2} \propto$ $L^{2}(2 \pi / L)^{2}$ in a $2 d$ system. In a one-dimensional (1d) system this proportionality is $L(2 \pi / L)^{2}$ and for $3 \mathrm{~d}$ it is $L^{3}(2 \pi / L)^{2}$. The energy of the fluctuation does not diverge with the length $L$ in $1 \mathrm{~d}$ and $2 \mathrm{~d}$ and, therefore, long wavelength fluctuations can be excited easily. This argument was generalized for lattice systems by Mermin and Wagner [26, 27]. In a 2d crystal the deviation of a particle diverges logarithmically: $\left\langle\left|\boldsymbol{u}(\boldsymbol{R})-\boldsymbol{u}\left(\boldsymbol{R}^{\prime}\right)\right|\right\rangle \propto \ln \left(\left|\boldsymbol{R}-\boldsymbol{R}^{\prime}\right|\right)$. Due to this relatively slow divergence crystals exist in $2 \mathrm{~d}$ but have quasi-long-range instead of true long-range translational order. The translational correlation function

$$
G_{G}(r) \propto\left\langle\sum_{j} \exp \left(\mathrm{i} \boldsymbol{G} \cdot \boldsymbol{r}_{j}\right)\right\rangle
$$

decays algebraically in the crystal phase. Here, $G$ is a vector of the reciprocal lattice.

Furthermore, computer simulations and $2 \mathrm{~d}$ colloidal model systems have shown that particles with crystal-like surroundings are present before freezing; the structural change at the transition is not as clear as in $3 d$ [28]. This is discussed in more detail in section 3.2. Also, there is strong evidence that many $2 \mathrm{~d}$ crystals do not melt in a firstorder transition but in two second-order transitions. This melting behavior is explained by a theory developed by Kosterlitz, Thouless, Halperin, Nelson and Young (KTHNY theory) $[29,30,17,31,32]$, which predicts that a third phase - the hexatic phase - with short-range translational and quasi-long-range orientational order exists between crystal and liquid. In triangular $2 \mathrm{~d}$ crystals the orientational order is measured by the correlation function

$$
\begin{gathered}
G_{6}(r) \propto\left\langle q_{6}(\boldsymbol{r}) q_{6}^{*}(0)\right\rangle, \text { where } \\
q_{6}\left(\boldsymbol{r}_{j}\right)=\frac{1}{N_{j}} \sum_{k} \mathrm{e}^{6 \mathrm{i} \theta_{j k}}
\end{gathered}
$$

is the $2 \mathrm{~d}$ orientational order parameter. $\theta_{j k}$ is the angle between $r_{j k}$ and an arbitrary fixed axis and $N_{j}$ is the number of nearest neighbors of particle $j$. The hexatic phase is also expected to influence the freezing transition, especially if the system is kept close to equilibrium during freezing. Clear 


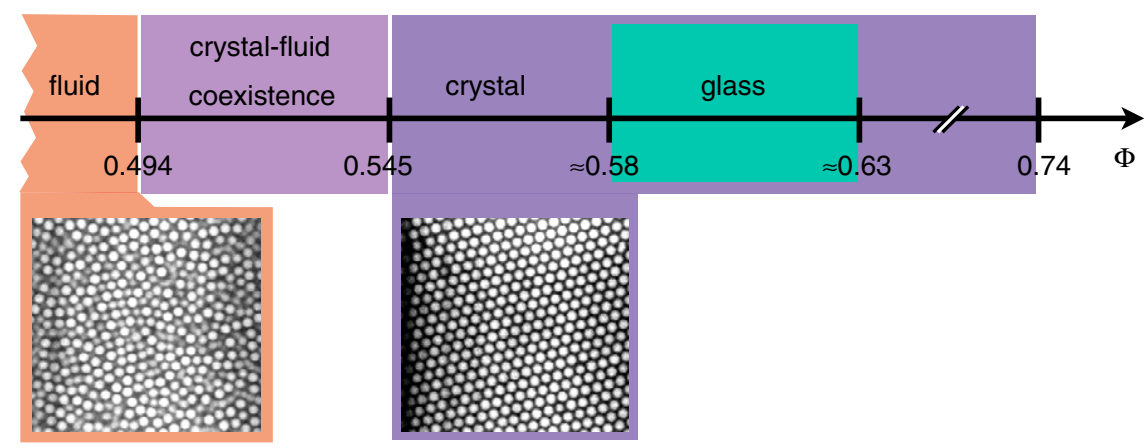

Figure 2. Phase behavior of HS particles depending on the volume fraction $\phi$. Images of HS-like PMMA particles illustrate the fluid and the crystal phase.

experimental evidence for melting of $2 \mathrm{~d}$ crystals according to KTHNY theory has been found during the last two decades; these findings and possible implications for freezing in $2 \mathrm{~d}$ are discussed in section 3 .

\subsection{Interactions and comparison with atomic systems}

A wide range of different crystal structures is observed in colloids, as different interactions such as hard sphere repulsion (section 2), electrostatic repulsion or attraction, electric and magnetic dipole-dipole interaction, van der Waals interaction or depletion interaction are all relevant under certain conditions. Some of them can be of the same order of magnitude, which leads to competition between different structures and complex phase behavior. Also, in many dense suspensions the interactions are not simply pairwise but manybody interactions are relevant [33], such as, for example, in metals. Furthermore, the interactions between colloidal particles can be tuned by tailoring the particles themselves; this can be achieved by using particles with special electric or magnetic properties, a special solvent or by applying an external electric or magnetic field [7].

Due to the good experimental accessibility and the possibilities for tailoring the interactions of the particles, colloidal suspensions are interesting model systems for fundamental studies of crystal nucleation and growth. Nevertheless, there are general differences between colloidal and atomic systems, which have atomic or molecular building blocks with typical size $\lesssim 1 \mathrm{~nm}$. As discussed in section 2.3, colloids are polydisperse to some extent and size distributions that are wider than $5 \%$ can have a strong effect on crystallization and phase behavior [34]. The free energy cost of the liquid-solid interface can be increased by polydispersity and, as a consequence, crystal nucleation is suppressed in $2 \mathrm{~d}$ and $3 \mathrm{~d}$ systems. In many cases a fractionation of large and small particles has to take place before nucleation can start. This can lead to subtle effects such as nucleation in several bursts [35]. Since colloidal particles are suspended in a solvent, the total kinetic energy of the colloidal particles is not conserved and the number of conserved quantities in colloidal and atomic systems is therefore not the same. The solvent also gives rise to long-range hydrodynamic interactions between the colloidal particles. The interaction energies between colloidal particles is of the same order of magnitude as the corresponding energies in atomic or molecular systems, but the density of colloidal particles is much lower due to their larger size. As a consequence, the specific heat of colloidal suspensions is usually of no importance, since the total interaction energy is small. Freezing and melting in colloidal systems happens virtually without a change of temperature and the diffusion of heat in the vicinity of the fluid-solid interface is, therefore, of no importance in colloidal crystallization.

\section{Hard spheres}

Due to the conceptual simplicity of their interaction, their phase behavior (see figure 2) and the importance of packing effects in many systems, hard spheres (HS) are an important model system in statistical mechanics. HS are the simplest system showing a phase behavior that is analogous to that of atomic substances. The crystallization of HS in $3 \mathrm{~d}$ was predicted by Kirkwood [24] and the first observations came from computer simulations [36, 37]. Since HS have no potential energy, their phase behavior is determined entirely by entropy [38] and depends only on volume fraction $\phi$. Fluid and crystal coexist between the freezing point $\phi_{\mathrm{f}}=0.494$ and the melting point $\phi_{\mathrm{m}}=0.545$ [39], while pure crystal is stable for $\phi>\phi_{\mathrm{m}}$. The configurational entropy of HS is decreased when they form a crystal lattice. This reduction of entropy is, however, more than compensated by an increase of entropy due to the larger free volume for local movements in the crystal lattice; space is used more efficiently in the crystal than in an disordered state. An intuitive understanding of this can be obtained by comparing the maximum volume fractions in a crystal and in a random particle configuration. In hexagonally close-packed crystals the maximum volume fraction is 0.74 , while it is reduced to 0.64 for a disordered particle configuration. At higher volume fractions a glass transition is observed at $\phi_{\mathrm{g}} \approx 0.58[40,41]$. However, for $\phi>$ $\phi_{\mathrm{g}}$ shear-induced crystals with typically plate-like or needlelike shapes can be observed. The formation of such crystals depends on the shear history of the sample and they form by heterogeneous nucleation typically on container walls [42]. Moreover, some samples with $\phi>\phi_{\mathrm{g}}$ have been found to crystallize in micro-gravity, while they remained glassy for at least a year under normal gravity [43]. 
HS systems can be realized experimentally with sterically stabilized particles in apolar solvents [40] and with charged colloids in solvents with high salt content, which results in a short screening length for the electrostatic interaction [44]. Sterically stabilized poly-methyl-methacrylate (PMMA) particles have been found to show a phase behavior that is remarkably close to that of hard spheres [40, 45-47]. With the proper scaling of the particle diameter in order to take the thickness of the surface layer for steric stabilization into account, these particles are found to freeze and melt at the volume fractions predicted by computer simulations [36]. The experimental freezing volume fraction found by Pusey and van Megen [40] can be reproduced in simulations, if the softness due to the layer of poly-hydroxystearic acid for steric stabilization and a small charge are considered. However, there are large discrepancies for the nucleation rates from experiment and simulation. In a more recent comparison of experimental and simulation data it is found that PMMA particles most probably carry a small charge, which seems to decrease when the density of the particles is increased [48]; the PMMA particles appear to be more HS-like at high volume fractions close to $\phi_{\mathrm{g}}$.

In general, HS crystals grow as a random stacking of hexagonally close-packed planes (rhcp) [40, 49-52], since the difference in free energy between the fcc and hcp lattice is so small that the conversion from rhcp stacking to fcc crystalthe structure with lowest free energy - takes $\sim 10^{3}$ times longer than the initial nucleation and growth of the crystal [53-56]. As a consequence, rapidly grown crystals tend to contain a large number of stacking faults (equal mixture of fcc and hcp), while slow growth results in crystals with a clear tendency towards fcc [49]. However, the amount of fcc-like stacking of the planes and the rate with which the crystal converts to the fcc stacking also appears to depend on the shear history of the sample [57].

\subsection{Homogeneous nucleation of HS crystal}

Numerous experiments have been carried out for measuring the nucleation rate (figure 3) and the growth laws in HS suspensions. Probably the most accurate determination of nucleation rates was done by Bragg scattering studies and small-angle light scattering (SALS). SALS is sensitive to largescale density fluctuations. Crystal nuclei are denser than the fluid around them due to Laplace pressure and, in the coexistence region, due to the larger density of the crystal. Therefore, crystal nuclei give rise to a peak at small scattering angles $[58,59,14,60]$. The position and the width of this peak can be used to determine the average distance and size of the nuclei. However, no detailed information about the structure and shape of the nuclei is obtained. By following the evolution of the small-angle peak, it was found that the mode of growth varies from diffusion limited to reaction limited and an intermediate behavior is observed in most cases. Timeresolved Bragg scattering gives detailed information on crystal growth [61-63, 43, 64]. Even information about the formation of crystal precursors can be obtained, although the crystals are too small to give rise to Bragg peaks at this stage; the formation of crystal precursors causes a change of the fluid peak in the

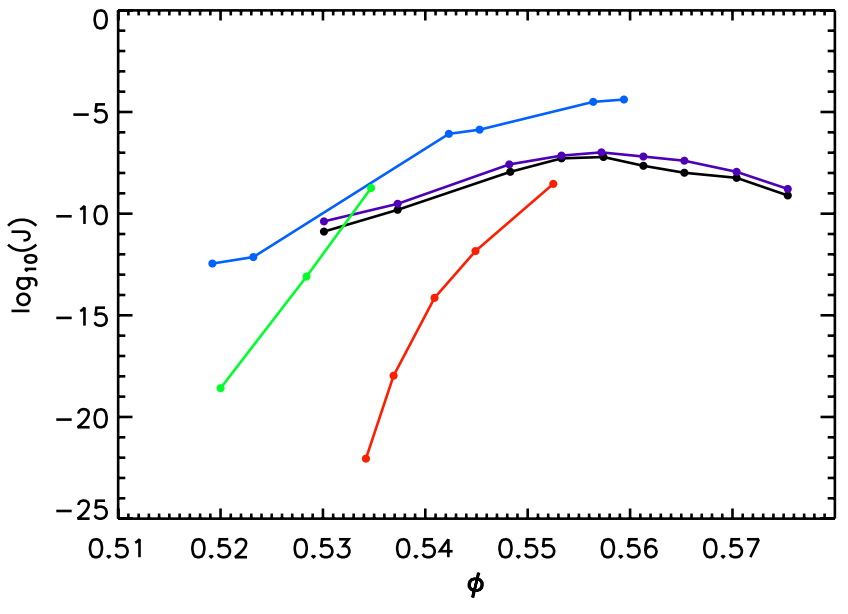

Figure 3. Nucleation rate density $J$ of hard spheres as a function of volume fraction $\phi$ observed in experiments ( $\bullet$ (purple) maximal nucleation rate density $J_{\max }$, average $\bullet$ (black) $J_{\text {av }}[63], \bullet$ (blue) [59]) and simulations $(\bullet$ (green) monodisperse HS, $\bullet$ (red) polydisperse HS, [34]).

scattering signal, which allows us to determine the number of crystal nuclei that form during the induction phase [65].

For $\phi_{\mathrm{f}}<\phi<\phi_{\mathrm{m}}$ the density difference between crystal and fluid is relatively large, especially for $\phi$ considerably smaller than $\phi_{\mathrm{m}}$ and for small nuclei, which are strongly compressed by Laplace pressure. Therefore, diffusion is expected to be important for the transport of particles from the fluid towards the crystallites. This diffusion-limited process is indeed observed for $\phi$ slightly larger than $\phi_{\mathrm{f}}[59,14]$, as the average size $L$ of the crystallites is found to typically grow as $L \propto t^{1 / 2}$. A depletion zone with a diameter of the order of the crystal diameter is expected to form around a crystal nucleus. For $\phi>\phi_{\mathrm{m}}$ the large difference of chemical potential between the fluid and the solid favors a fast insertion of spheres into the crystal and the density difference between fluid and crystal is not as large as for $\phi<\phi_{\mathrm{m}}$. This situation corresponds to the reaction-limited regime with $L \propto t$. These observations are well reproduced by a model for classical crystal growth $[14,15]$ and a density functional theory approach [66]. The occurrence of the reaction-limited regime and growth exponents $>1 / 2$ indicate that there are factors that limit the importance of diffusion. This is expected to be the case when the incorporation of particles into the crystal is so slow that diffusion is never rate limiting or, for $\phi>\phi_{\mathrm{m}}$, when structural relaxation from fluid to crystal is so fast that the density field has no time to respond to the pressure field. The first case is expected to be relevant for polydisperse suspensions. However, these two scenarios are hardly fulfilled in experiments with monodisperse colloids (polydispersity $<5 \%$ ). Two further reasons why growth exponents $>1 / 2$ can occur are a decrease of the density difference as crystals grow with time and the finite width of the liquid-crystal interface, which is not obtained with simple theories giving a growth exponent of $1 / 2$ [66].

As shown in figure 3 , the homogeneous nucleation rate density $J$ is found to grow with increasing $\phi$-increasing 
undercooling - until it reaches its maximum at $\phi \approx \phi_{\mathrm{m}}$. For higher $\phi, J$ decreases and vanishes at the glass transition at $\phi_{\mathrm{g}} \approx 0.58$. The maximum of the nucleation rate close to $\phi_{\mathrm{m}}$ is usually explained by a change of the kinetics of crystal growth. The chemical potential difference $\Delta \mu$ grows monotonically when $\phi$ is increased and, accordingly, the nucleation rate is also expected to grow. The maximum of the nucleation rate is then attributed to a decrease of the rate with which particles can be incorporated into nuclei with a volume fraction $\phi_{\mathrm{m}}<\phi<\phi_{\mathrm{g}}$. Hydrodynamic interactions leading to viscous loss are one reason for this decrease of $J$ beyond $\phi_{\mathrm{m}}$ [67]. However, in micro-gravity, HS have been found to crystallize at volume fractions even higher than $\phi_{\mathrm{g}}$, which suggests that kinetic factors cannot be the only reason for the maximum of the nucleation rate density $J$. This issue was addressed by MC computer simulations of polydisperse HS at fixed $\Delta \mu$ [68]. Surprisingly, the simulations show that the height $\Delta G^{*}$ of the free energy barrier as a function of $\Delta \mu$ has a minimum in the range $0.6<\Delta \mu / k_{\mathrm{B}} T<1.1$. This is in contrast to Turnbull's suggestion that the surface energy $\gamma$ should be proportional to $\Delta H$, the latent heat of fusion [11]. This minimum can be understood if a dependence of $\gamma$ on $\Delta \mu$ is taken into account, which can be approximated as $\gamma=\gamma_{0}(1+\alpha|\Delta \mu|)$. Thus, the assumption of CNT that $\gamma$ is a constant is not correct for HS. Furthermore, the simulations suggest that the kinetic factor of crystal growth - the probability with which a critical nucleus crosses the barrier and begins to grow-varies by at most an order of magnitude. Therefore, the decrease of the nucleation rate with increasing supersaturation is dominated by the increase of $\gamma$ and not by a slowing down of the kinetics. This could be tested experimentally: the distribution of the final average crystallite sizes from crystallization experiments conducted at different undercooling (different $\phi$ ) is expected to be proportional to $\exp \left(\Delta G^{*} /\left[4 k_{\mathrm{B}} T\right]\right)[68,69]$. As a consequence, a minimum in the average crystallite size at some volume fraction corresponding to the minimum of $\Delta G^{*}$ should be observable. Also, an increase of the crystallite size at high supersaturations beyond the maximum of $J$, at $\phi>\phi_{\mathrm{m}}$, would indicate that the increase of $\gamma$ with $\Delta \mu$ is real.

The nucleation rate is generally determined by the volume fraction of the supercooled fluid. However, it is also timedependent and a complex interplay between the forming nuclei and the surrounding fluid is not uncommon. Accelerated nucleation has been found for volume fractions $\phi>\phi_{\mathrm{m}}$ [62]. Since the crystal nuclei at such high densities have a volume fraction that is larger than the average volume fraction of the whole sample, the volume fraction must decrease in the regions between nuclei during nucleation. This leads to an increase in the nucleation rate $J$, because $J$ increases when $\phi$ decreases towards $\phi_{\mathrm{m}}$.

During the growth of crystals in micro-gravity, the formation of dendrites has been observed in suspensions of monodisperse HS by direct imaging with a camera [43, 70, 64]. Dendritic growth has not been observed under normal gravity conditions, even in density matched samples. The critical nucleus radius for the onset of the dendritic growth instability was estimated to be $R_{\text {crit }} \approx 70 \sigma$, where $\sigma$ is the particle diameter. Furthermore, Bragg scattering data obtained in micro-gravity at $\phi=0.528$ suggest that interactions between growing crystallites can be important in HS [64]. As expected at this high $\phi$, the average size $L$ of the nuclei was observed to grow as $L \propto t$ while the number of crystallites decreased and the crystal volume fraction increased as $t^{2.2}$, which is significantly slower than the expected $t^{3}$ behavior. This suggests that small nuclei are incorporated by large ones even before the end of the nucleation phase. However, such behavior might depend on specific conditions such as polydispersity.

Time-resolved SALS and Bragg scattering experiments have provided a lot of insight into the nucleation and growth processes and are a testbed for accurate models of crystal nucleation and growth. For example, it has become clear that crystal growth is typically limited by diffusion for $\phi<\phi_{\mathrm{m}}$ and by the incorporation of particles into the crystallites for $\phi>\phi_{\mathrm{m}}$. However, these experiments have not yielded detailed information on nuclei of critical size, since such small nuclei do not generate Bragg peaks and, furthermore, the scattering signals of nuclei with different ages and sizes are averaged in scattering experiments. Very small nuclei can, however, be studied directly by real-space imaging such as DIC microscopy [71] or confocal microscopy [72, 73] and in computer simulations.

\subsection{Critical nuclei}

The properties of small crystal nuclei are of particular interest. They are not well known but are of prime importance for the nucleation process and for the early stage of crystal growth. One reason for the failure of CNT to explain measured nucleation rates is the assumption that small crystal nuclei have the same properties as the bulk crystal, e.g. the structure of the nuclei is assumed to be that of the bulk crystal and no dependence of the surface tension $\gamma$ on the size of the nuclei or on $\Delta \mu$ is taken into account. In HS, crystal nuclei are usually approximated as spherical clusters, while aspherical shapes are found in experiments and simulations [74, 75]. However, the shape of critical nuclei strongly depends on the particle interaction and the results presented below should be expected to differ in systems with other interactions. For example, in a remarkable study of crystal nucleation in apoferritin protein [76], nuclei of planar shape have been observed. Planar nuclei are also expected to play a central role in nucleation of short rods, as presented in section 4.

Since the formation of a critical nucleus in a supercooled liquid is a rare and short-lived event, it is difficult to catch it in experiments. Biased MC simulations have the advantage that such events of interest can be made to occur more often. Therefore, much of the current information about the formation of critical nuclei comes from simulations. In a series of biased MC simulations, nuclei of given sizes are produced and their free energies are determined, while the kinetic factors giving the transition probability from one nucleus size to another are determined by kinetic MC or molecular dynamics (MD) simulations. In this way the properties of nuclei of different sizes as well as the nucleation rate density $J$ can be determined. This has been applied for a study of HS [68] and $\gamma$ has been found to depend on volume fraction or, equivalently, 

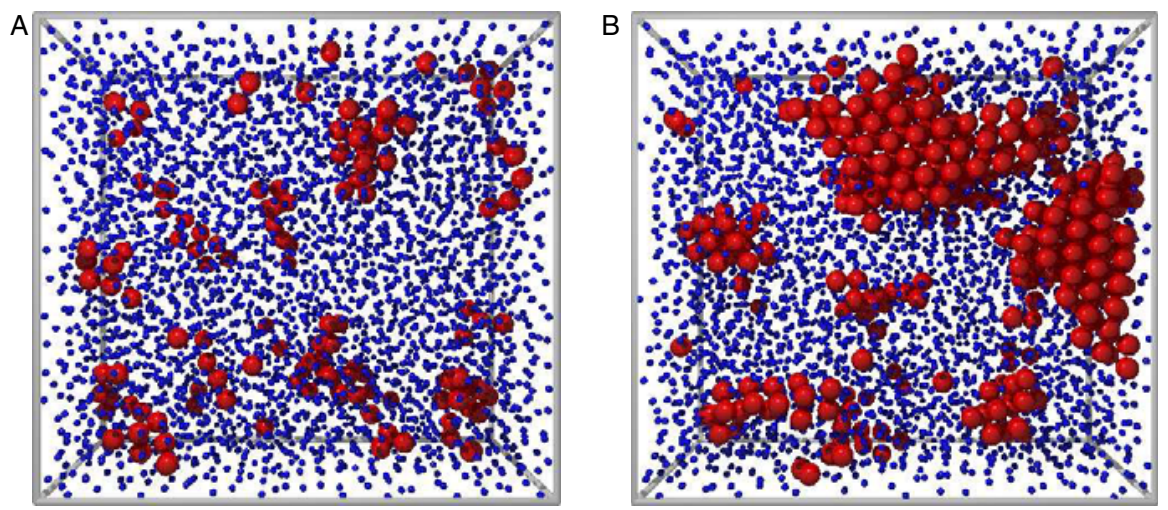

Figure 4. Crystal nuclei of slightly charged PMMA particles observed by confocal microscopy $(\phi=0.414)$. Particles in a crystal-like surrounding are represented by red spheres that are drawn to scale, while the particles in the fluid state are shown by smaller blue spheres to enhance the visibility of the crystalline regions. (A) 15 min after shear melting: subcritical nuclei have formed from structural fluctuations in the metastable fluid. (B) After 58 min: two postcritical nuclei are observed in addition to subcritical ones.

supersaturation. This is important, since $J$ depends sensibly on its value. The structure of the critical nucleus was determined with the bond-order parameter method $[77,78]$ and the dominating structure was found to be rhcp.

Some of these simulation results can be compared with a real-space imaging study of the early phase of homogeneous nucleation in dense suspensions of weakly charged PMMA spheres with $\phi_{\mathrm{f}}=0.41$ and $\phi_{\mathrm{m}} \approx 0.46$ [74]. Confocal microscopy was used to determine a number of key quantities for crystal nucleation by determining accurate particle coordinates and by following the evolution of the local structure with a local bond-order parameter. Close to the maximum of the nucleation rate density $J$ at $\phi \approx$ 0.47 , the evolution of a large number of crystal nuclei could be followed and the size of the critical nucleus could be determined from the condition that subcritical nuclei shrink on average, while postcritical nuclei grow (figure 4). Furthermore, the surface tension $\gamma$ was estimated in two ways. For the smallest subcritical nuclei the positive surface energy dominates and, as expected, their number is found to decrease with increasing surface area. From the statistics of these nuclei $\gamma \approx 0.027 k T / a^{2}$ is obtained. The second value, $\gamma_{\mathrm{CNT}} \approx$ $0.075 k_{\mathrm{B}} T / a^{2}$, is determined from nucleation rates according to CNT [79]. Due to the small charge of the particles, this value for $\gamma$ is about a factor of two smaller than the value expected from computer simulations and theoretical calculations for HS [68, 80-82]. A similar reduction of $\gamma$ has been found from computer simulations of slightly charged spheres [83]. The difference between the two experimentally determined values for $\gamma$ suggests that the properties of nuclei vary as they grow from subcritical to postcritical size. The structure of small crystal nuclei was analyzed by the same bond-order parameter method as used in the simulations of [83]. The crystal nuclei were found to be predominantly rhcp, which is the structure expected for the bulk crystal. No contribution of bcc-like order was found, although this is suggested by a mean-field argument [84]. Generally, the nuclei have a rough surface in confocal snapshots, presumably due to their low surface tension $\gamma$.
In a recent confocal microscopy study of homogeneous nucleation in HS a similarly low value of $\gamma=0.5 \pm$ $0.05 k_{\mathrm{B}} T / a^{2}$ and rough surfaces were found for small crystal nuclei [85]. Furthermore, the experimentally determined nucleation density rates could be explained quantitatively, when the irregular structures of the nuclei were taken into account in $\Delta G$ (equation (1)) in terms of a configurational entropy contribution. This new contribution to $\Delta G$ might resolve the issue of large differences between nucleation rate densities found in experiments and those expected from theory and simulations, as shown in figure 3 .

More details on the structure of critical nuclei in HS were obtained from long MD simulation runs with 32000 and 65000 particles, in which 10 critical nuclei were observed [75]. The structure was analyzed with a combined method of local bond-order parameters and a modified Voronoi construction. Some of the observed nuclei are shown in figure 5. As expected, the nuclei contain stacking faults; but, in addition, heavily faulted structures, a twinned morphology and even fivefold axes have been observed. As in the confocal microscopy study mentioned above, the average stacking was close to rhcp, but the stacking varied strongly from nucleus to nucleus. The interfaces of the nuclei were found to be diffuse and the overall shape was aspherical. The volume fraction of the nuclei at the induction time was 0.58 , which is comparable to the value measured during crystallization of hard sphere colloids by light scattering [63]. The growth did not proceed through the addition of planes but was found to be located at stacking faults or twinning planes. Growth at such defects is commonly observed in many metallic particles [86]; it was found to be concentrated at crossing stacking faults. Such sites were previously identified to favor fcc over hcp [87].

\subsection{Polydispersity}

The nucleation and growth of colloidal crystals can depend strongly on the polydispersity of the particles. For different types of particles, polydispersity in size, charge, magnetic moment or other properties can be of relevance. Here, an overview of the influence of size polydispersity $\sigma$ in $\mathrm{HS}$ is 


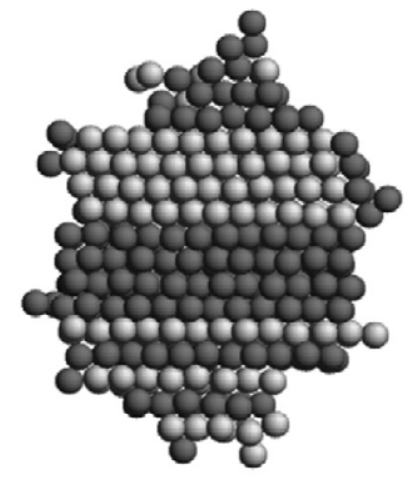

(a)

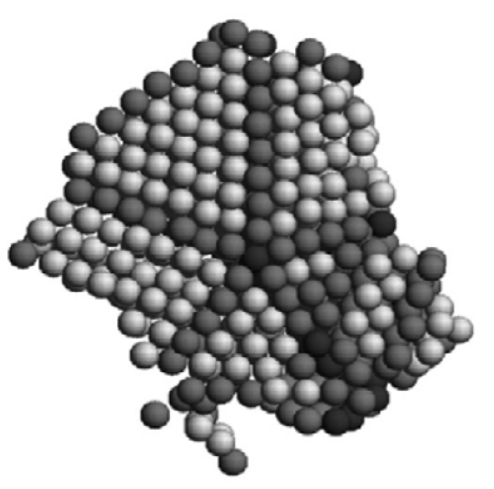

(c)

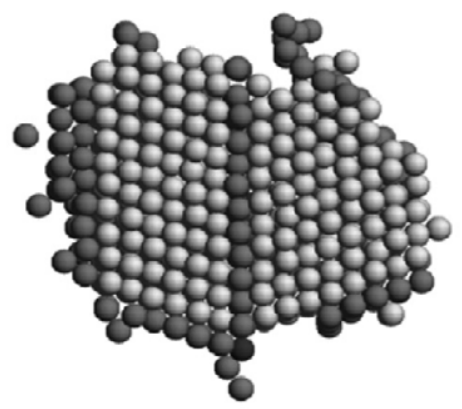

(e)

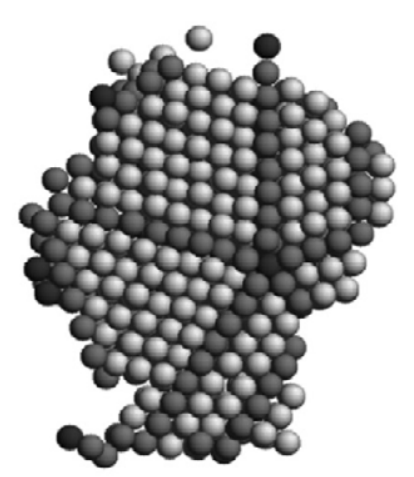

(b)

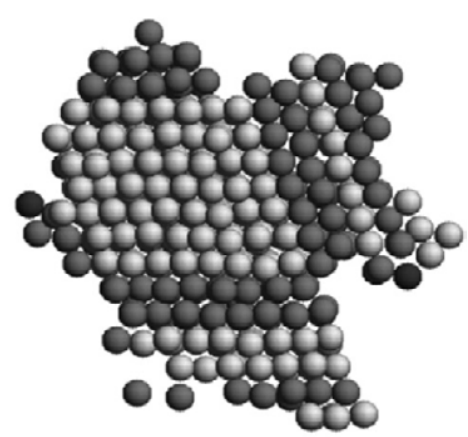

(d)

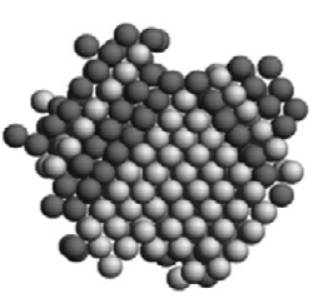

(f)

Figure 5. Reprinted figure with permission from [75]. Copyright 2003 by the American Physical Society. Cross sections through several nuclei at the induction time. The lighter colored particles are fcc while the darker colored particles are hcp ordered. The near-black colored particles (located along the fivefold axes in (b) and (c)) possess a local twisted icosahedral environment. For clarity only particles with these three ordered local structures are shown. The observed structures are: (a) a single dominant stacking direction, ((b), (c)) multiply twinned nuclei with fivefold axis, (d) mainly fcc order with stacking faults, ((e), (f)) nuclei with mostly fcc order bounded by hcp planes or stacking faults.

given, which is defined as the standard deviation of the radius distribution divided by the mean radius:

$$
\sigma=\sqrt{\left\langle r^{2}\right\rangle-\langle r\rangle^{2}} /\langle r\rangle .
$$

For particles with unimodal size distribution, computer simulations show a changed phase behavior and crystal nucleation for polydispersities $>5 \%$ [34]. The coexistence region of fluid and crystal is shifted to higher volume fractions $[44,88]$ and crystallization is observed up to a polydispersity of $12 \%$. However, fractionation of small and large particles takes place such that the polydispersity of the crystal does not exceed 5.7\%. At fixed supersaturation $\Delta \mu$, the height of the nucleation barrier $\Delta G^{*}$ is not affected by polydispersities up to $5 \%$, while for larger polydispersities the barrier increases rapidly. This must be due to an increase of the surface tension $\gamma$ with polydispersity and it is in agreement with the observation that crystallization is suppressed for polydispersities larger than $12 \%$ [34].

However, polydispersity is not just limiting crystal nucleation; its effect is rather complex and depends on the detailed size distribution. The addition of a small amount of smaller HS to a monodisperse suspension of large HS was found to considerably retard crystallization [89], which 
changes crystallization from diffusion limited to interface limited with increasing polydispersity. Two exemplary cases have been studied by light scattering in systems of monodisperse HS particles with an additional fraction (1-3\%) of larger or smaller particles [90]; the size ratio of the small and large particles was $\alpha=r_{\mathrm{B}} / r_{\mathrm{A}}=0.82$. The crystallinity (fraction of the sample that gives rise to Bragg scattering), the position of the first Bragg peak and the average crystal size were determined. The time until rapid crystal growth did set in was prolonged in both cases. With additional larger particles, the crystal was found to have essentially the same properties as in a monodisperse sample, but the lattice constant was moderately increased and larger crystal grains formed. This suggests that the larger particles are fractionated out of the crystal to a large extent. In the case of a small fraction of smaller particles, a slow initial growth process was observed that lasted about 10 times longer than the induction time in monodisperse samples. These results suggest that a small amount of larger particles decreases the density of nucleation sites, which results in fewer large crystals, while the density of nucleation sites is not reduced when smaller particles are admixed. Similar results were obtained in a study of crystal growth in HS with a polydispersity of $4.8 \%$ [35]. The crystallization process was followed by measuring the Bragg reflections from suspensions with $0.51<\phi<0.57$; the crystal structure factor was measured and the time-dependent crystallinity $X(t)$, volume fraction of the crystal $\phi_{\text {xtal }}(t)$, average linear grain size $\langle L(t)\rangle$, number of crystallites $N_{\text {xtal }}(t)$ and the nucleation rate density $J(t)$ were extracted from the data. The crystallization process could be divided into three phases. During the first phase, the induction time $J(t)$ is found to be high and slowly decreasing due to the formation of a large number of crystal precursors that have neither liquid nor crystal structure and, therefore, do not give rise to Bragg peaks. $X(t)$ remains very low during this phase. During the second phase the number of crystal precursors decreases by about one order of magnitude, while $\langle L(t)\rangle$ begins to grow and $\phi_{\text {xtal }}(t)$ drops to an intermediate value. The drop in $N_{\text {xtal }}(t)$ coincides with the appearance of a second Bragg peak and is followed by a considerable increase of $X(t)$. The mode of growth-merging of several crystal precursors or growth of a subset of the precursors - could not be identified from the data. During the third phase, $J(t)$ reappears in a second, short burst of nucleation; $\langle L(t)\rangle$ and $N_{\text {xtal }}(t)$ grow rapidly before they plateau at the end of this phase and $\phi_{\mathrm{xtal}}(t)$ reaches its final value. The nucleation in two steps is due to polydispersity; during the first nucleation event fractionation of the particles occurs and nucleation halts when the polydispersity in the remaining fluid becomes too large. However, the fractionation continues and a second nucleation event can occur. This suggests that stepwise nucleation might be a universal feature in polydisperse systems.

A high polydispersity is usually associated with glass formation and the absence of crystallization. However, the formation of ordered structures is not necessarily suppressed by a high polydispersity. This has been shown in a light scattering study of HS with bimodal but continuous size distribution and average size ratio $\alpha=0.57$ [91,92]. In spite of this large value, one Bragg peak was observed approximately two days after shear melting the sample, indicating that the particles form a partially ordered plane-like structure. The observed Bragg scattering is compatible with a planar structure, where the particle distance within a layer is larger than that in a hexagonal layer and the distance between planes is decreased. This structure is motivated by the possibility that smaller particles can sit on interstitial sites between planes formed by large particles. There is no long-range order within the planes and the order perpendicular to the planes is also reduced; thus, just one Bragg peak is observed. After shearing the sample for two days by shaking it, the Bragg peak appeared to be split into two peaks, the old, nucleated Bragg peak and a new peak at somewhat smaller scattering vector $q$. This second peak is explained by a partial fractionation during shearing, which leads to the formation of crystallites that contain mainly larger particles. Thus, a high polydispersity does not necessarily prevent structural order but strongly influences the crystal structure and the nucleation process.

\section{Freezing and melting in two dimensions}

A deeper understanding of the phase transitions between liquid and crystal in $2 \mathrm{~d}$ has been obtained during the last three decades from theoretical and experimental studies of crystal melting. This work has been motivated by KTHNY theory developed in the 1970s by Kosterlitz, Thouless, Halperin, Nelson and Young [93, 30, 29, 31]. Since the work on the melting transition is also important for the understanding of crystallization in $2 \mathrm{~d}$, the basic ideas and the most important experimental results confirming the KTHNY theory are presented in section 3.1 and work on the freezing transition is discussed thereafter in section 3.2. A detailed review of KTHNY theory and related experiments is given in [32]. Next to KTHNY, other scenarios for melting in $2 \mathrm{~d}$ have been studied. The formation of grain boundaries [94-96], which appear as chains of dislocations and condensation of dislocations [97], have been proposed. Whether KTHNY theory or another melting mechanism applies, depends on the core energy of a dislocation $[94,95]$ but to date there is no clear rule for the validity of the different theories.

\subsection{Melting of two-dimensional crystals according to KTHNY theory}

According to KTHNY theory, melting of $2 d$ crystals is due to the formation of dislocations, which are topological defects. Since only pairs of dislocations can appear spontaneously, melting must be due to the unbinding of two bound dislocations (figures 6(A) and 7) that form just before or during the melting process. Unbound dislocations destroy the quasi-long-range translational order, which becomes short-range (exponential decay), but the orientational order is less affected. It is reduced from long-range in the crystal phase to quasi-longrange (figure $6 \mathrm{~B}$ ). The resulting phase with short-range translational and quasi-long-range orientational order is the hexatic phase, which is specific for $2 \mathrm{~d}$ systems and separates the isotropic liquid and the crystal. The transition from the 


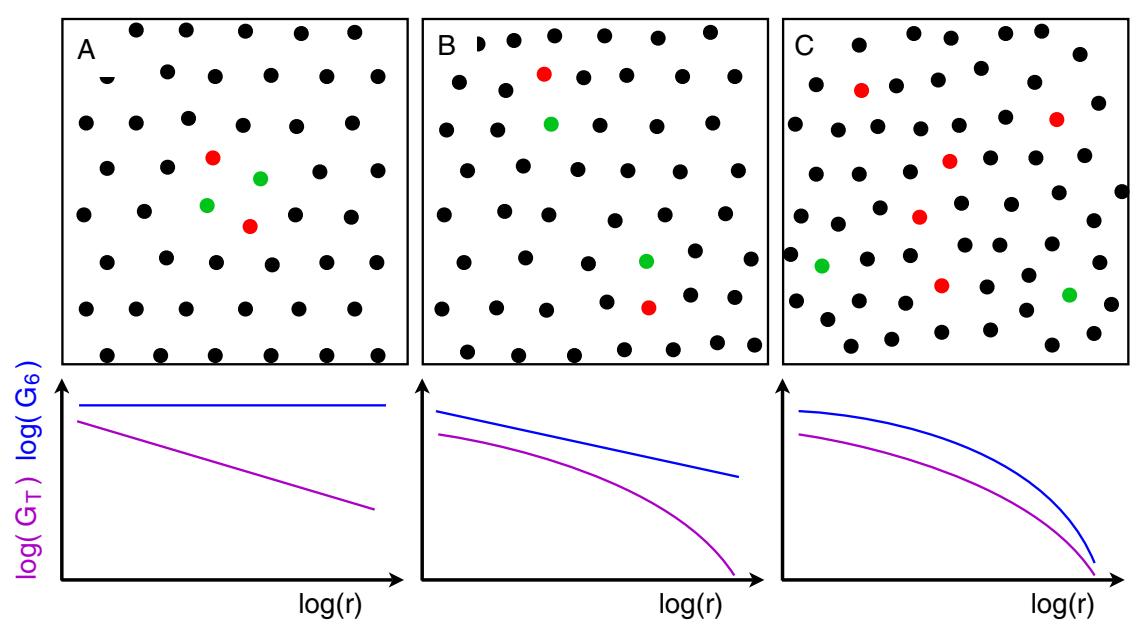

Figure 6. Overview of topological defects in two dimensions: (A) a bound pair of dislocations in a triangular crystal, (B) two unbound dislocations in the hexatic phase and (C) disclinations in the isotropic liquid phase. Sevenfold and fivefold coordinated particles are shown by - and $\bullet$, respectively. The corresponding behaviors of the bond-order correlation function $G_{6}(r)$ (blue) and the translational correlation function $G_{\mathrm{T}}(r)$ (purple) are shown below.

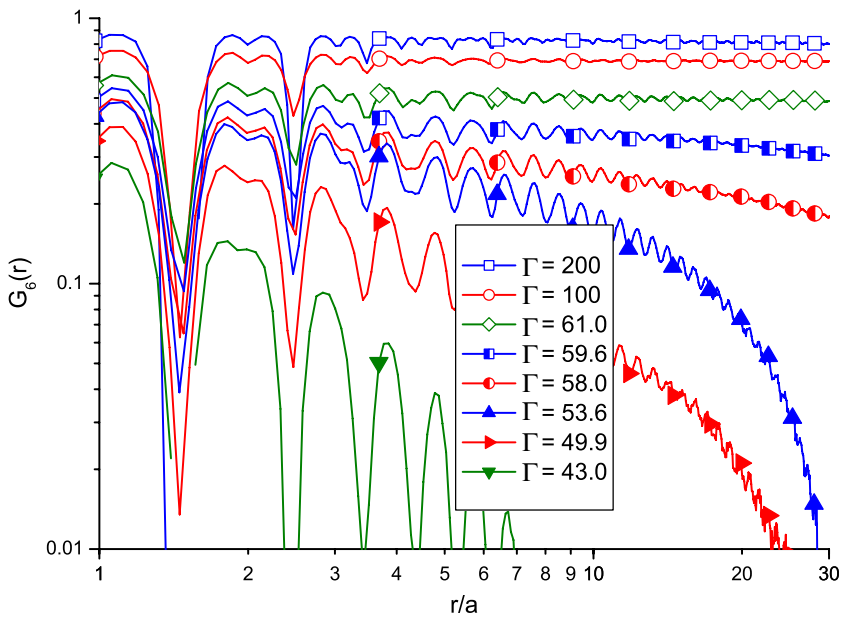

Figure 7. Reprinted figure with permission from [109]. Copyright 2007 by the American Physical Society. Orientational correlation function $G_{6}(r)$ as a function of the inverse temperature in a log- $\log$ plot. From top to bottom: three curves for the crystalline phase showing the long-range orientational order $\left(\lim _{r \rightarrow \infty} G_{6}(r) \neq 0\right)$, two curves showing the quasi-long-range order of the hexatic phase $\left(G_{6}(r) \propto r^{-\eta_{6}}\right)$ and three curves showing the short-range order of the isotropic liquid $\left(G_{6}(r) \propto \mathrm{e}^{-r / \xi_{6}}\right)$.

hexatic to the liquid happens at a higher temperature-or lower concentration - than the melting transition. Dislocations unbind into disclinations, another type of topological defect. This unbinding reduces the orientational order from quasilong-range to short-range (figure 6(C)). Thus, KTHNY theory predicts three equilibrium phases-liquid, hexatic and crystal-and two second-order transitions, which means that no fluid-crystal or hexatic-crystal coexistence regimes are expected.

Initially, the validity of KTHNY theory was mainly tested with computer simulations [98-100]. However, due to the limited number of simulated particles no clear results for or against KTHNY were obtained. More recent computer simulations show the behavior expected from KTHNY [101, 102]. In experiments, the hexatic phase was first found by Murray and van Winkle [103] in a system of charge-stabilized polystyrene particles in between two glass plates. Further observations followed from a system of essentially hard spheres in $2 \mathrm{~d}$ [104], where the crystal-hexatic and hexatic-liquid transitions were observed but appeared to be of first order, and from particles with electric dipoledipole interaction $[105,106]$. The clearest evidence for the validity of KTHNY theory has been obtained with a system using super-paramagnetic particles with magnetic dipoledipole interaction $[18,107]$. As illustrated in figure 7 , both the translational and the orientational correlation function show the expected behavior and allow us to locate the crystalhexatic and the hexatic-liquid transition [18]. The most precise results are obtained from the time-dependent orientational correlation function $G_{6}(t)$, which can be determined with better accuracy, because the finite size of the observed volume is not limiting the accuracy of the result. The clearest evidence that the transitions do indeed happen due to the unbinding of dislocation pairs and dislocations into two disclinations according to KTHNY has been given by showing that the transition temperatures are those predicted by the theory [108, 109]. The unbinding of the same topological defects is also relevant in $2 \mathrm{~d}$ granular systems, which can exhibit an analogous phase behavior although they are far from equilibrium [110].

\subsection{Crystallization in two dimensions}

While clear evidence for the $2 \mathrm{~d}$ melting scenario according to KTHNY theory has been obtained, crystal nucleation in $2 \mathrm{~d}$ is not as well studied and there are important unresolved issues. Although two second-order transitions are expected from KTHNY, the nature of the freezing transition is still an unresolved question. Especially for hard discs, there are indications for a first-order transition [101]. For systems that 
(a)

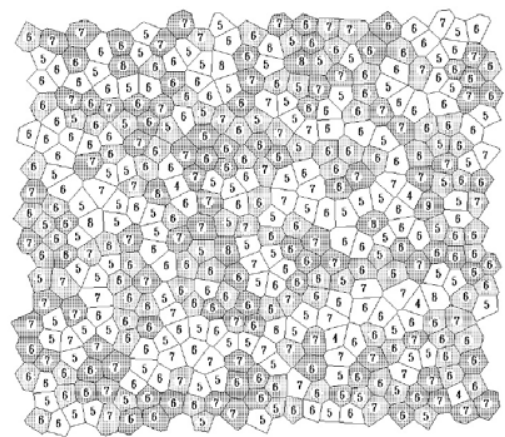

(b)

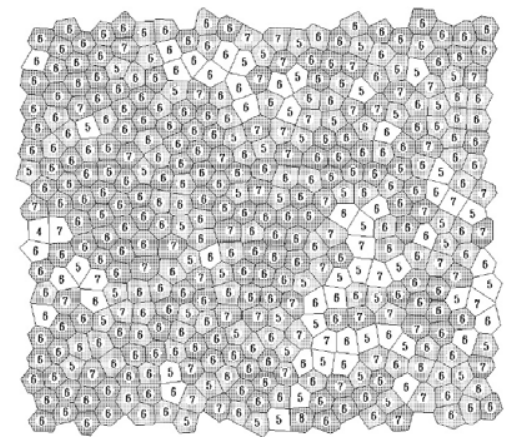

(c)

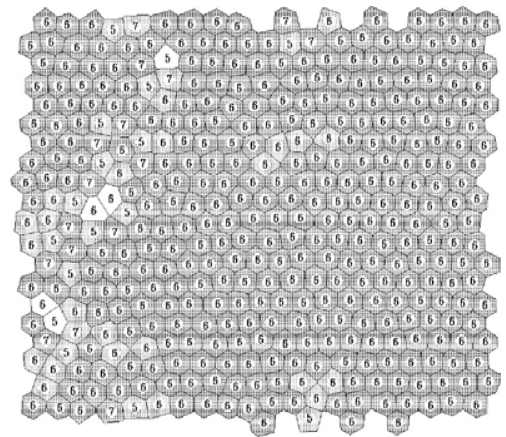

Figure 8. Reprinted figure with permission from [28]. Copyright 2005 by the American Physical Society. Snapshots of configurations at packing fractions $\phi=0.5$ (a), $\phi=0.65$ (b) and $\phi=0.7$ (c), with Voronoi cells. Darker cells correspond to polygons of class A $(\zeta \in(1.108 ; 1.159))$, lighter cells to polygons of class B $(\zeta \in(1.159 ; 1.25))$ and the numbers indicate the type of polygon (number of vertices).

show melting according to KTHNY it is not clear under which conditions the hexatic phase is of importance for freezing. Furthermore, the structural changes during freezing are not as clear as in 3d [28], which can complicate the experimental determination of both the temperatures where freezing and melting occur and the differentiation between the formation of the hexatic phase and a liquid-crystal coexistence region as expected for a first-order freezing transition.

In several studies, $2 \mathrm{~d}$ liquids just below freezing have been observed to contain crystal-like patches of sixfold coordinated particles [111, 28, 112, 105], which might be expected from the changes in $g(r)$ just before crystallization (see section 1.2 and [22]). The existence of crystal-like patches in the liquid complicates the detection of the freezing transition and it shows that, close to the freezing transition, the liquid is more complex than suggested by the pair-correlation function $g(r)$. The importance of higher correlations has been shown in a microscopy and simulation study of a $2 \mathrm{~d}$ liquid [23]. An accurate description of the experimentally observed liquidcrystal phase behavior has also been investigated by density functional theory (DFT) for a $2 \mathrm{~d}$ liquid with repulsive dipoledipole interaction $[113,114]$. Two- and three-point particle correlations have to be taken into account to obtain a free energy functional that agrees well with experimental results. The information given by $g(r)$ is, therefore clearly not sufficient for a detailed understanding of freezing. The DFT approach is, however, in some respects a rough approximation for true $2 \mathrm{~d}$ crystals. A perfect triangular crystal with longrange translational order is assumed. The liquid-crystal transition is, therefore, of first order and dislocation pairs, the typical defects seen both in experiment and simulations [115], are not obtained.

For a clearer separation of the structural properties of the liquid and the crystal phase, a structural shape factor $\zeta=$ $C^{2} /(4 \pi S)$ has been introduced [28], which can be applied when the particle coordinates are known as in simulations or microscopy studies. $\zeta$ relies on a Voronoi analysis, which allows us to determine the circumference $C$ and the surface area $S$ of the Voronoi cell of each particle. In MC simulations of hard discs it was found that $\zeta$ reflects the structural changes close to melting in more detail than the changes in $g(r)$. As illustrated in figure 8, three classes of particles were defined according to $\zeta<1.159$ (class A), $1.159<\zeta<1.25$ (class B) and $1.25<\zeta$ (class C), where the limit between A and $\mathrm{B}$ is motivated by a minimum in the histogram of $\zeta$ that is observed just before freezing and class $\mathrm{C}$ is chosen such that it is virtually absent in the crystal. Thus, in a crystal close to freezing mostly class A is observed, while just above the transition patches of mainly $A$ as well as mixed patches of $\mathrm{B}$ or $\mathrm{C}$ are observed. The $\zeta$ parameter is apparently useful for getting a picture of ordered and disordered domains and, therefore, should also be applied in studies of crystal melting, although, in an experimental study of the freezing transition [116], $\zeta$ alone was not sufficient for a clear differentiation of crystal and liquid.

As the localization of the freezing transition from structural changes is not straightforward, changes of the particle fluctuations at freezing can give important additional information. In the liquid, fluctuations are strong and rather uncorrelated, while they are clearly correlated in the crystal. This is reflected by the stability of the local structure in the crystal and its relatively fast decay in the liquid. Thus, a dramatic change of fluctuations is expected at freezing; an analysis of the freezing transition based on both typical structures and fluctuations just above the freezing temperature has been proposed [112]. Two typical structures consisting of seven particles were analyzed. $\Gamma_{6}$ represents a sixfold coordinated particle as expected in the crystal and $\Gamma_{5}$ corresponds to a fivefold coordinated particle and an additional neighboring particle; this typically represents a dislocation. For both $\Gamma_{6}$ and $\Gamma_{5}$ it is assumed that the particles show independent Gaussian fluctuations and the properties of each are thus described by a distribution of the local bond-order parameter $q_{6}$ and by the width of the Gaussian fluctuations. The values for both parameters were determined from MC simulations of hard discs and the Lennard-Jones liquid (LJ). The freezing transition was located by checking for the suitability of the $\Gamma_{6}$ and $\Gamma_{5}$ structure to represent the order of the system. Indeed it is found that the significance level of the representation drops quite sharply at the concentration or temperature where freezing is expected. From this analysis of hard discs and the LJ liquid the concentration of crystallike sixfold coordinated particles at freezing is expected to be 
rather high, with $50-56 \%$ of all particles, and at the melting point it was found to be in the range of $75-80 \%$. Moreover, a Lennard-Jones criterion for $2 \mathrm{~d}$ was obtained: at freezing the root-mean-square fluctuations were found to be in the range $0.12 a-0.13 a$, where $a$ is the nearest-neighbor distance.

The conditions under which the hexatic appears during freezing have not yet been clarified. The $2 \mathrm{~d}$ system with magnetic dipole-dipole interaction [18, 107, 109] has been used to study crystallization and the hexatic was observed under very slow cooling [109], while it was absent for cooling with larger quenches from the liquid towards the crystal phase [116]. Experiments with shallow gradual quenches would be of interest to clarify when the hexatic appears.

\section{Crystal nucleation of short, rod-shaped particles}

Most work on crystallization in colloidal systems focuses on spherical particles, although there are important examples of non-spherical particles, e.g. in clays [117]. Here, recent work on dense crystal structures of ellipsoidal particles and on nucleation in short rods is summarized. The latter highlights the difficulties that are encountered with nonspherical particles. Work on the ordering of rods in liquid crystalline phases and other structures has been summarized in [118].

As for hard spheres, the most favorable structure of hard ellipsoids is the one that allows the densest packing. However, for ellipsoids this structure is unknown. Structures based on the fcc lattice have been proposed: when an fcc lattice with spherical particles is stretched in an arbitrary direction, a lattice with ellipsoidal particles and the same volume fraction is obtained [119]. That denser structures are possible has been shown recently [120]. Ellipsoids of revolution with aspect ratio of length and diameter $L / D \approx 3$ can reach a packing of $\phi=0.7707$ with two particles per unit cell that are tilted with respect to each other. A related monoclinic structure with two particles per unit cell was studied with MC simulations [121] and, starting from the fcc-like lattice mentioned above, it was found to form spontaneously for ellipsoids of revolution with $3<L / D<6$. Ellipsoids with $L / D<3$ were found to remain in the fcc-like structure. These observations, however, do not show that this monoclinic, tilted structure is formed spontaneously by ellipsoids that are initially in the nematic or a liquid phase. Thus, nucleation of such elongated particles is largely unknown.

From liquid crystals it is well known that a suspension of rods passes through a nematic phase at intermediate concentrations before reaching the crystalline state. However, short rods with an aspect ratio $L / D \leqslant 3$ crystallize directly without intermediate nematic order. The details of this phase transition are not well understood and experimental results on the nucleation process and crystal growth are scarce. A real-space imaging investigation [122] revealed a transition from the fluid to smectic order in rods with $L / D=3.5$. Initially, a single layer that grew laterally was observed and additional layers only formed after substantial growth of the first one. Smectic ordering and no nematic phase was found for $4.4 \leqslant L / D \leqslant 8.0$. For longer rods, $10 \leqslant L / D \leqslant 35$, ordering proceeds via the nematic phase.

The experimental observations agree with results obtained from simulations, but the theoretical understanding and also the computer simulations are hampered by the fact that the nucleation process in rods differs from that for spherical particles. Therefore, the appropriate order parameter for a description of the fluid-crystal transition is unknown [123-125]. Biased MC simulations have been used to study the nucleation process in a box containing 2400 spherocylinders with aspect ratio $L / D=2$. A combination of a maximum surface-to-surface distance and a maximum value for the angle formed by the long axes of neighboring particles was used as an approximate order parameter to identify particles in a crystal-like surrounding. Large ordered clusters with up to 80 particles were obtained in the simulations, but the vast majority of the particles were arranged in a single layer of rods. Although the conditions were chosen such that the crystal is stable, the formation of a second layer was not observed, since rods lying flat on the surface of the first layer obstruct this process. The reorientation of these rods to an upright position on the layer is an improbable process and the growth of additional layers is, therefore, hindered for kinetic reasons. The one-layer nucleus is 'poisoned' by rods lying flat on it. Since the free energy of a single layer grows monotonically with its size, it does not cross the nucleation barrier and, therefore, cannot form a stable crystal. In the simulations, the 'poisoning' rods can be pre-aligned by choosing a bias that favors rods that stand upright on the layer. With this method the formation of a second layer was observed, but it was found to be an additional nucleation event. Therefore, the consecutive formation of layers is a very improbable nucleation pathway and nucleation could not be observed in the simulations, since no adequate reaction coordinate for following the crystallization pathway is known. Similar problems were encountered in a simulation study of crystal nucleation of ellipsoids of revolution with an axis ratio $a / b=1.25$ [125]. Nucleation in rods might involve the fusion of two or more layer-shaped subcritical nuclei. It is expected that an external field for pre-aligning the rods would greatly enhance the nucleation rate. The observation of a layer agrees qualitatively with the experimental results in [122].

\section{Soft repulsive and attractive interactions}

\subsection{Crystal-crystal coexistence}

A liquid only appears in the phase diagram of a substance, if there is a large enough attractive range in the interaction of the pair potential [126-130]. When the range of the attraction, $\delta$, is decreased, the critical temperature $T_{\mathrm{c}}$ is lowered and for $\delta / \sigma \approx 1 / 3$ it reaches the triple point temperature $T_{\mathrm{t}}$ and the liquid phase becomes metastable. Here, $\sigma$ denotes the diameter of a colloidal particle. An analogous situation occurs in systems with a very narrow attractive range, $\delta / \sigma \leqslant 0.07$. A dense and an expanded crystal phase with the same structure can coexist [131]. The transition from one to the other phase is first order and there is a crystal-crystal critical point in the 
phase diagram. This situation was explored by MC simulations for particles with an HS core and an attraction due to a square well potential [132]. In this system, the internal energy is lowered abruptly when the density is increased such that the majority of the nearest-neighbor distances is reduced to a value $<\sigma+\delta$, where $\delta$ is the width of the square well potential. When the temperature is low enough, this reduction of internal energy can outweigh the penalty due to the entropy decrease caused by the higher density. A cell-model calculation yields the relation $\rho_{\mathrm{c}} / \rho_{0}=(\delta / \sigma+1)^{-3}$ for the critical density $\rho_{\mathrm{c}} ; \rho_{0}$ is the density at close packing. This result was corroborated by MC simulations that show a weak dependence of $T_{\mathrm{c}}$ on $\delta$ and suggest that for $\delta / \sigma<0.06$ the crystal-crystal transition should be observed; for larger $\delta$ the expanded crystal phase becomes metastable. Such a crystal-crystal transition is expected to be observable for systems of uncharged colloids with a depletion interaction that is sufficiently short-ranged.

The influence of a crystal-crystal coexistence on the crystallization process was studied in more detail by MC computer simulations of HS with depletion interaction due to smaller hard spheres [133]. The size ratio of small and large spheres was $\alpha=0.1$ (0.05) and, correspondingly, the expanded crystal is metastable (stable). Simulations were carried out at two volume fractions of the small particles, $\phi_{\mathrm{s}}=0.05$ and 0.1 . For $\phi_{\mathrm{s}}=0.05$ the effective temperature is above the critical point of crystal-crystal coexistence and there is only one crystal phase. The nucleation process is in good agreement with CNT, as the free energy barrier is well described by the CNT expression and the density of the crystal nuclei is the same as the density of the bulk crystal and does not depend on the number $n$ of particles in the nucleus. At $\phi_{\mathrm{s}}=0.1$, the effective temperature is below the critical temperature and there is a metastable expanded crystal phase. The density of the nuclei is found to increase with $n$, which is in contrast to both CNT (the density is always that of the bulk crystal) and the droplet model (the density decreases with increasing $n$ because of Laplace pressure). This counterintuitive behavior can be explained by the fact that the pressure inside a crystal nucleus depends on both Laplace pressure and the surface stress $[134,135]$. The latter can have a large effect when the compressibility is large, which is the case near a metastable crystal phase. Thus, the observed behavior is a direct consequence of the proximity of a crystal-crystal critical point.

On the experimental side, hints of the effect of a metastable crystal state have been found in a video-microscopy study of the sublimation of wall crystals in colloids with depletion interaction [136]. With a size ratio $0.003<\alpha<$ 0.014 the range of the attraction was very small. Metastable wall crystals have been found to sublimate faster into the gas phase as soon as they shrink below a critical size of $n=$ $25 \pm 5$ particles. If the melting process was governed by the surface, the evaporation rate should be proportional to $n^{1 / 2}$ and evaporation should become slower when the crystallites shrink. However, these crystallites undergo accelerated sublimation by transforming into a dense liquid, which has a slightly lower density than the crystal and evaporates quickly. This accelerated melting is reminiscent of a coexistence of a dense and an expanded crystal phase. In this particular case, however, the expanded crystal phase probably does not form because of the polydispersity $\approx 3.5 \%$ of the colloidal spheres used. This example probably shows how an intervening metastable phase can have a profound effect on the kinetics of a phase transition.

\subsection{Soft particles}

A lot of insight into crystal nucleation and growth has been obtained from systems based on HS. But during recent years, soft particles realized by, for example, star polymers or microgel particles have gained a lot of attention for applications [137-140] and as model systems. Poly $(N-$ isopropylacrylamide) (PNIPAM) microgels are the most common thermo-responsive microgel particles [141]. Their phase behavior is expected to be richer than that of HS [142, 143]. With thermo- or pH-responsive microgel particles the effective volume fraction can be varied easily by changing the size of the particles. Thus, very fast density quenches and, furthermore, very high densities that require a compression or interpenetration of the particles can be studied. Moreover, a change from the compressed to the swollen state of the particles allows us to study the transition from HS-like to soft particles in a single experimental system [144, 145]. With UV-visible spectroscopy measurements the crystallization kinetics of aqueous suspensions of PNIPAM particles was followed at different temperatures corresponding to volume fractions $0.51<\phi<0.56$ [145]. In the swollen state, the kinetics of the crystallinity, the number of crystals and the average size of the crystals was found to be close to results obtained with HS in micro-gravity [64]. This is not too surprising, since the PNIPAM particles contain a large amount of water; they almost match the density of the solvent and no sedimentation of crystals was observed. In the shrunken state the kinetics becomes much slower, which must be related to the changed interaction and is not understood in detail. An interesting decrease of the freezing point of PNIPAM particles with decreasing effective volume fraction has been determined by real-space imaging [146] but detailed information about crystallization was not obtained.

A theoretical study of charged soft particles [143] indicates that the common criteria for freezing and melting do not hold for super-soft particles and, furthermore, at high concentrations the structures are expected to differ from those of HS-like systems. The phase behavior of very soft particles with uniform charge was studied with a genetic algorithm for free energy minimization. The crystal structures that are expected for increasing particle concentration are fcc and bcc and, at very high densities, which force the particles to either shrink or interpenetrate, hexagonal, body-centered orthorhombic (bco) and simple trigonal (tri). For the highest densities, it is found that both the Hansen-Verlet and the Lindemann criterion do not hold. For example, the Lindemann criterion would favor fcc or bcc at very high concentrations. Furthermore, the crystal is found only for sufficiently charged particles such that the charge repulsion between the particles is strong enough to give rise to structural order. To date, however, in experiments only fcc or rhcp order has been found in neutral and charged microgel suspensions [147-149]. 


\section{Binary crystals}

Binary colloidal crystals were first observed by Sanders $[150,151]$ in gem opals, which consist of dried silica particles. The first laboratory-grown binary colloidal crystals consisted of charged latex particles $[152,153]$. While the stoichiometry of binary crystals in atomic systems is controlled by the charges of the ions forming the crystal, this is not a strict restriction in colloidal crystals. Indeed, an $\mathrm{AB}_{8}$ structure without an atomic analog has been observed [154]. Here, A denotes the large and $\mathrm{B}$ the small colloidal particles. The crystal structures formed in mixtures of small and large colloidal particles are of interest for applications such as photonic crystals [3] and also due to the analogy with atomic binary crystals. Compared with monodisperse colloidal suspensions, the size difference between large and small particles can facilitate the formation of crystals with good long-range order, which is often desired for applications. A lot of progress is currently made in understanding the phase behavior and in controlling the structures in binary colloidal suspensions. However, the crystallization process is not well studied.

\subsection{Binary hard sphere crystals}

The surprisingly complex phase diagram of binary HS was mapped out with light scattering experiments with various size ratios $\alpha=r_{\mathrm{B}} / r_{\mathrm{A}}$ and number ratios $n_{\mathrm{B}} / n_{\mathrm{A}}$. Hexagonal $\mathrm{AB}_{2}$ (space group $P 6 / \mathrm{mmm}$ ) and cubic $\mathrm{AB}_{13}$ (space group $F m \overline{3} \mathrm{c}$ ) were observed $[155,156] . \mathrm{AB}_{2}$ was found at a size ratio of $\alpha=0.58$ and hints for it were found at $\alpha=0.61$. Since crystal growth in binary samples is slow, sedimentation can be a problem. Therefore, samples are usually prepared with solvents that closely match the density of the particles and, in addition, the samples can be tumbled slowly to average out sedimentation effects. Interestingly, the complex $\mathrm{AB}_{13}$ structure was found to grow faster than $\mathrm{AB}_{2}$ crystals; it is speculated that this is related to the high degree of local icosahedral order in $\mathrm{AB}_{13}$, which might lead to a low free energy of formation for crystallites with this structure.

When the number density of one species dominates, pure A or B crystals coexist with a liquid phase. For intermediate number ratios, coexistence of $\mathrm{AB}_{13}$ and liquid $\left(9 \leqslant n_{\mathrm{B}} / n_{\mathrm{A}} \leqslant 16\right)$ as well as $\mathrm{AB}_{2}$ and liquid $\left(4 \leqslant n_{\mathrm{B}} / n_{\mathrm{A}} \leqslant 6\right)$ are observed. According to these regions of crystal-liquid coexistence, three eutectic regions - each lying between two of the crystal-liquid coexistence regions-are expected and two of them, $\mathrm{B}$-crystal- $\mathrm{AB}_{13}$-liquid and $\mathrm{AB}_{13}-\mathrm{AB}_{2}$-liquid, have been observed experimentally. The third eutectic region, $\mathrm{A}-$ crystal- $\mathrm{AB}_{2}$-liquid was not found, because the evolution of the samples was very slow at high concentrations of large particles and they remained amorphous for several months. Bartlett et al calculated a phase diagram using semi-empirical equations of state $[157,29]$ and the assumption that the two species are completely miscible in the liquid state but not miscible in the solid state. This phase diagram does not predict $\mathrm{AB}_{2}$ or $\mathrm{AB}_{13}$ structures, but for the cases where one species dominates it agrees well with experimental results. A first prediction for the stability of different structures can be obtained by comparing their volume fractions at close packing. As a rule of thumb, the structure that allows the highest volume fraction is the best for maximizing entropy; at volume fractions below close packing, this structure gives the particles the most space for local movements, which increase entropy. According to this criterion, the $\mathrm{AB}_{13}$ and $\mathrm{AB}_{2}$ structures are expected to be found in the range $0.5<\alpha<0.8$ [158]. In a more rigorous analysis of the stability of $\mathrm{AB}_{2}$ and $\mathrm{AB}_{13}$, the Gibbs free energy of $\mathrm{AB}_{2}$, $\mathrm{AB}_{13}$, pure fcc of $\mathrm{A}$ or $\mathrm{B}$, and liquid were determined [159] and the results were found to compare well with the experimental observations of Bartlett et al $[155,156]$. A cell theory approach was also used to determine the stability ranges of several binary structures [160]; the results for $\mathrm{AB}_{2}$ and $\mathrm{AB}_{13}$ agree well with those of [159].

For size ratios $\alpha<0.5$, theoretical and simulation results $[160,161]$ suggest that structures analogous to $\mathrm{NaCl}$ or NiAs are stable. $\mathrm{AB}$ crystals with $\mathrm{NaCl}$ structure have indeed been found for $\alpha=0.39$ and $n_{\mathrm{B}} / n_{\mathrm{A}}=1\left(\phi_{\mathrm{B}} / \phi_{\mathrm{A}}=\right.$ 0.06) [162]. The large A particles form an rhcp lattice and the small B particles sit in the interstices. The symmetry of the crystal is thus the same as for a pure crystal of A particles and, as a consequence, the powder Bragg pattern of the AB crystal is hard to distinguish from that of pure A crystal. However, the arrangement of the small B particles in the interstices was confirmed by laser scanning confocal microscopy.

At large size ratios $\alpha$ and $n_{\mathrm{B}} / n_{\mathrm{A}} \approx 1$ crystals with stoichiometry $\mathrm{AB}$ such as the $\mathrm{CsCl}$ structure are expected. The simple packing argument suggests that this structure is metastable, since its volume fraction at close packing is $0.729<\phi_{\text {fcc }}$. Nevertheless, it was found in a binary mixture with $\alpha=0.736$ and $n_{\mathrm{B}} / n_{\mathrm{A}} \approx 1$ by light scattering [163]. The samples were left undisturbed for several months and were not tumbled. As a consequence of slow sedimentation crystallites typically appeared in narrow, horizontal bands in the sample containers. However, the disappearance of the crystals in some samples after about a year indicates that the $\mathrm{CsCl}$ structure is indeed metastable.

Binary colloidal crystals have significantly different crystallization kinetics than monodisperse colloidal crystals. Relatively little is known about both nucleation and growth, since differential sedimentation of large and small particles complicates the study of this behavior. The crystallization kinetics of two crystallizing samples was studied by light scattering [165] in a micro-gravity environment on the International Space Station, where the deleterious effects of gravity are absent [164]. In one sample with $n_{\mathrm{B}} / n_{\mathrm{A}} \approx$ 4.7 and $\alpha=0.4, \mathrm{AB}_{6}$ crystals with bec structure formed, while $\mathrm{AB}_{13}$ crystals formed in the other with $n_{\mathrm{B}} / n_{\mathrm{A}} \approx 19$ and $\alpha=0.57$ (see figure 9). After the long induction times of typically $40 \mathrm{~h}$, the Bragg peaks of both crystal structures grow rapidly for $10-20 \mathrm{~h}$ and the width of the peaks decreases to significantly smaller values than in monodisperse HS crystals. This underlines the high degree of long-range order in these crystals. The long induction times are probably due to large-scale particle rearrangements that are necessary for the formation of nucleation sites. Especially in the case of the $\mathrm{AB}_{13}$ sample this is a probable scenario, since the stoichiometry of the whole sample is quite far from that of 

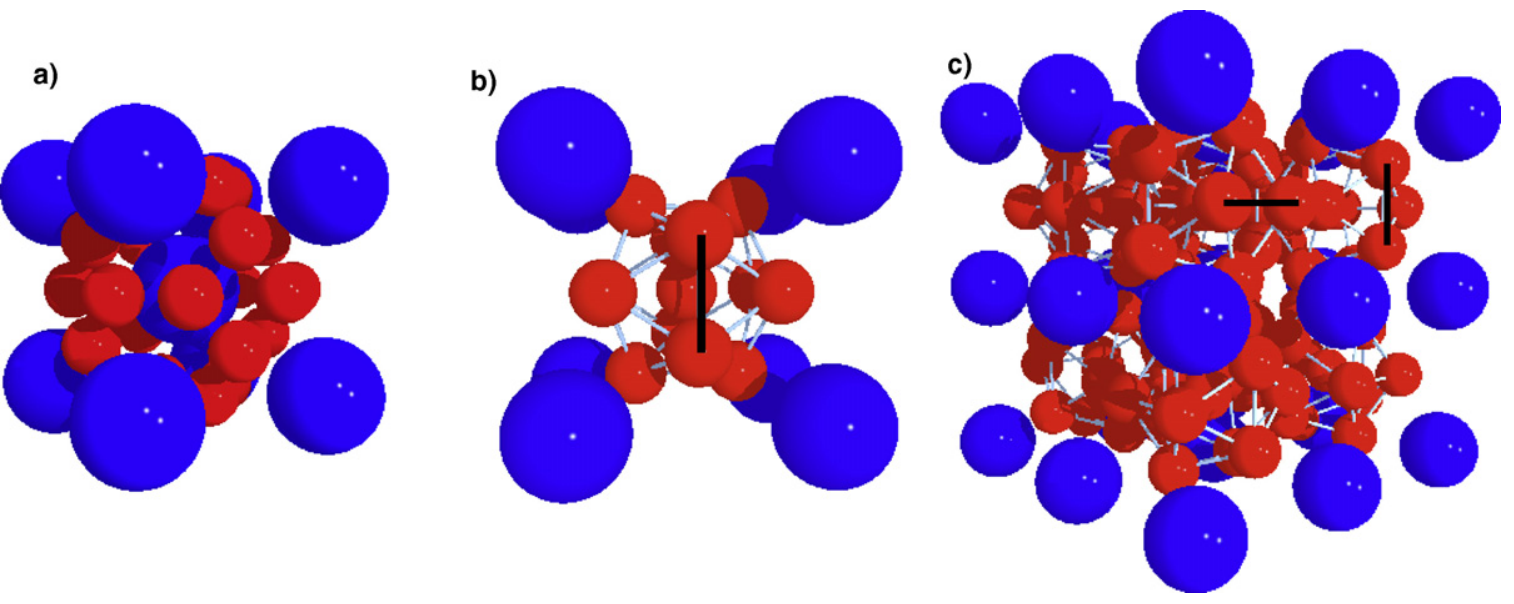

Figure 9. Unit cell structures of (a) $\mathrm{AB}_{6}$ and ((b), (c)) $\mathrm{AB}_{13}$ crystals observed in [164]. (b) shows the structure of $1 / 8$ of the unit cell. The small B particles are arranged in icosahedra surrounded by the large A particles forming a simple cubic lattice. (c) shows the unit cell of $\mathrm{AB}_{13}$. The changing orientation of the B icosahedra is illustrated by the black bars.

the crystal. The data obtained from the period of fast growth shows clear differences between the two samples. While in the case of $\mathrm{AB}_{13}$ virtually all nucleation sites appeared to form before the phase of fast growth, for $\mathrm{AB}_{6}$ a behavior expected close to the steady state nucleation and growth regime was observed. The large difference between the number ratios in the suspensions and the stoichiometry of the crystals is rather surprising; whether this is of particular importance for the formation of the crystals is an open question.

\subsection{Binary crystals of charged particles}

In contrast to HS, structures with relatively low volume fractions can be obtained with charged particles, where the contribution of the (screened) Coulomb interaction to the free energy is more important than entropy. When the Debye screening length is much larger than the radii of the particles, the stoichiometry of the binary lattice must be such that the charges of the particles compensate each other. However, for higher salinity of the solvent the screening length is so short that the stoichiometry is not determined by the charges. The whole spectrum of stoichiometries and structures ranging from charge-controlled to packing-controlled can be explored in colloidal suspensions, as the salinity of the solvent and, therefore, the screening is adjustable.

A large diversity of lattices has been observed in binary suspensions of sterically stabilized nanoparticles made of $\mathrm{Au}$, $\mathrm{Pb}, \mathrm{PbSe}, \mathrm{PbS}, \mathrm{Pd}, \mathrm{Fe}_{2} \mathrm{O}_{3}, \mathrm{CoPt}_{3}$ or $\mathrm{Bi}$ with a typical size of $\sim 10 \mathrm{~nm}$ [166]. As shown in figure 10, the observed structures range from $\mathrm{NaCl}$ to $\mathrm{AB}_{13}$ with almost every stoichiometry in between. The nanoparticles carry a small charge $(-e, 0, e$ or $2 e$ ), which can be influenced by coating the particles with various surfactants. As expected for charged particles, the size ratio of the particles is not as important as in hard spheres. A large variety of superlattices is also favored by the fact that some particles can occur in two charge states in the same suspension (e.g. neutral and $+e$ ). As a consequence, several different lattices have been observed to nucleate in one sample.
A structure such as $\mathrm{AB}_{13}$ with many particles of one species is preferred when the B particles are neutral.

Structures formed by oppositely charged particles can also be realized with poly-methyl-methacrylate (PMMA) particles. In a solvent containing cyclo-heptyl-bromide (CHB) these particles slowly change and even reverse their charge due to an increase of $\mathrm{Br}$ ions as a consequence of illumination with light [167]. In binary samples at a size ratio $\alpha=$ 0.93 , three crystal structures were observed. When the particles are close to neutral, substitutionally disordered rhcp crystals formed. With increasing charge, $\mathrm{CsCl}$ and $\mathrm{NaCl}$ structures with volume fraction $\phi=0.67$ and 0.49 were observed, respectively. The transition to $\mathrm{NaCl}$, a considerably less dense structure, is clearly a consequence of a growing electrostatic interaction between the particles; the observed transitions can be explained, at least partly, with the random primitive model [168]. In another study of binary PMMA suspensions [154], crystals with the stoichiometries $\mathrm{AB}, \mathrm{AB}_{6}$ and $\mathrm{AB}_{8}$ were identified. With a number ratio $n_{\mathrm{B}} / n_{\mathrm{A}}=4$, $\phi=0.23$, and a screening length $\sim 1.5 \mu \mathrm{m}, \mathrm{AB}$ crystals with $\mathrm{NaCl}$ and $\mathrm{NiAs}$ structures were found to coexist. For a size ratio $\alpha \approx 1$, crystals with $\mathrm{CsCl}$ structure formed. Surprisingly, for $\alpha \approx 0.31(\phi \approx 0.11)$ and number ratio $n_{\mathrm{B}} / n_{\mathrm{A}}=8$ the formation of $\mathrm{AB}_{6}$ crystals with face-centered orthorhombic structure was observed, as shown in figure 11 . The observations of the $\mathrm{NaCl}$ and $\mathrm{CsCl}$ structures at $\alpha \approx 1$ as well as the $\mathrm{AB}_{6}$ and $\mathrm{AB}_{8}$ structures for $\alpha \approx 0.31$ are in good agreement with calculations of Madelung energies and expectations from computer simulations $[154,169]$. An $\mathrm{AB}_{4}$ structure is also expected but was not observed experimentally. The similarity of the CsCl-like crystals observed with slightly charged particles [154] and the structures observed in [170] suggests that the particles in the latter study were also slightly oppositely charged.

One of the motivations for studying binary colloidal crystals are potential applications as optical bandgap materials. Large bandgaps can be obtained with diamond-like lattices $[171,172]$. A possible route to such crystals with diamond or pyrochlore structure is shown in a simulation study 

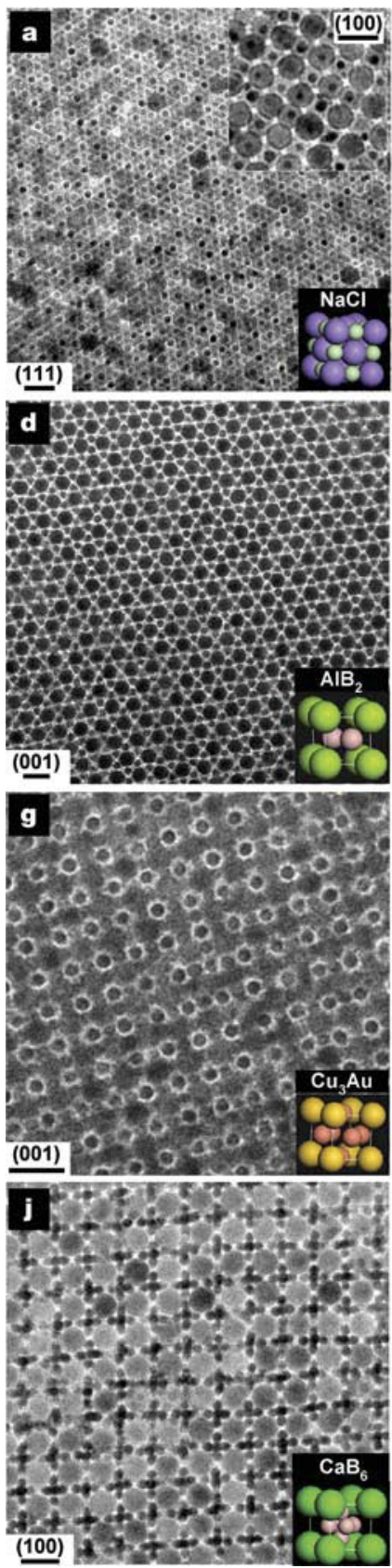
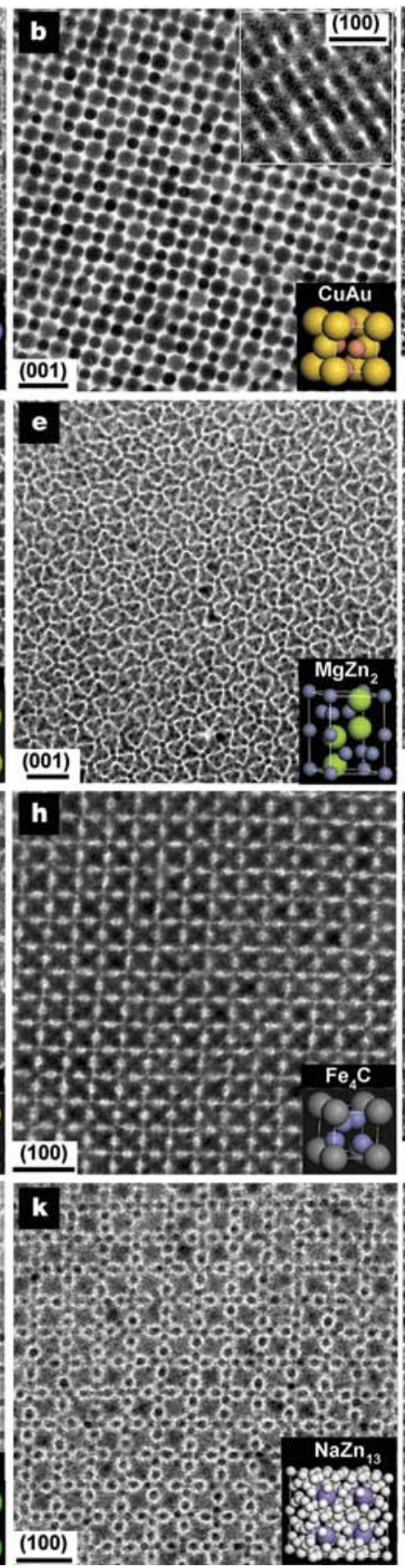
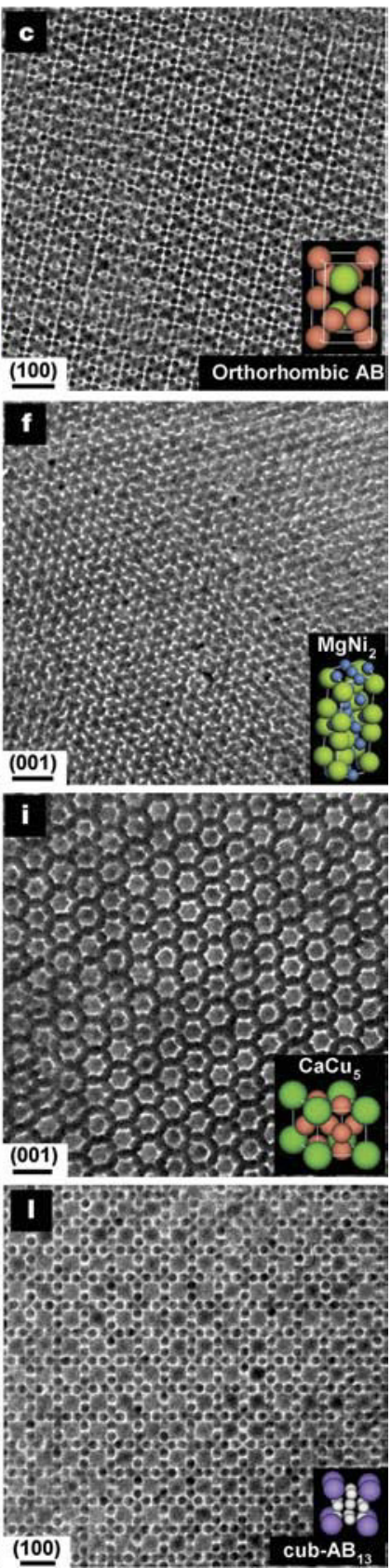

Figure 10. Reprinted with permission from Nature Publishing Group from [166]. TEM images of the characteristic projections of binary crystals, self-assembled from different nanoparticles, and modeled unit cells of the corresponding $3 \mathrm{~d}$ structures. The crystals are assembled from (a) g- $\mathrm{Fe}_{2} \mathrm{O}_{3}$ and $\mathrm{Au}$; (b) PbSe and $\mathrm{Au}$; (c) PbSe and $\mathrm{Pd}$; (d) PbS and Pd; (e) PbSe and Pd; (f) PbSe and Pd; (g) PbSe and $\mathrm{Ag}$; (h) PbSe and Pd; (i) PbSe and Au; (j) PbSe and Pd; (k), PbSe and Ag; (1) PbSe and Pd nanoparticles. Scale bars: (a), (b), (c), (e), (f), (i), (j), (k), (l), $20 \mathrm{~nm} ;(\mathrm{d}),(\mathrm{g}),(\mathrm{h}), 10 \mathrm{~nm}$. The lattice projection is labeled in each panel above the scale bar.

in [4]. MC simulations and free energy calculations show that in binary colloidal suspensions with size ratios $0.74<\alpha<$ 0.82 the Laves phases $\mathrm{MgCu}_{2}, \mathrm{MgZn}_{2}$ and $\mathrm{MgNi}_{2}$ coexist with fcc crystals of the large and small particles; the large particles in the Laves phases form the diamond-like lattice that is of interest for applications. Two of these Laves phases have been found in binary suspensions of nanoparticles [166]. Since all three have roughly the same free energy, a mixture is expected in experiments. In the simulations, the $\mathrm{MgCu}_{2}$ structure could be preferred by adding a templated surface, which favors its growth. Experiments along this route towards a diamond structure will be of broad interest.

\section{Crystallization on substrates and in external fields}

An applied field or a substrate, giving rise to an external potential that is larger or of the same order of magnitude as the inter-particle interaction, is expected to have a large effect on the phase behavior of colloidal suspensions. If the strength and, for example, the periodicity of an external field 

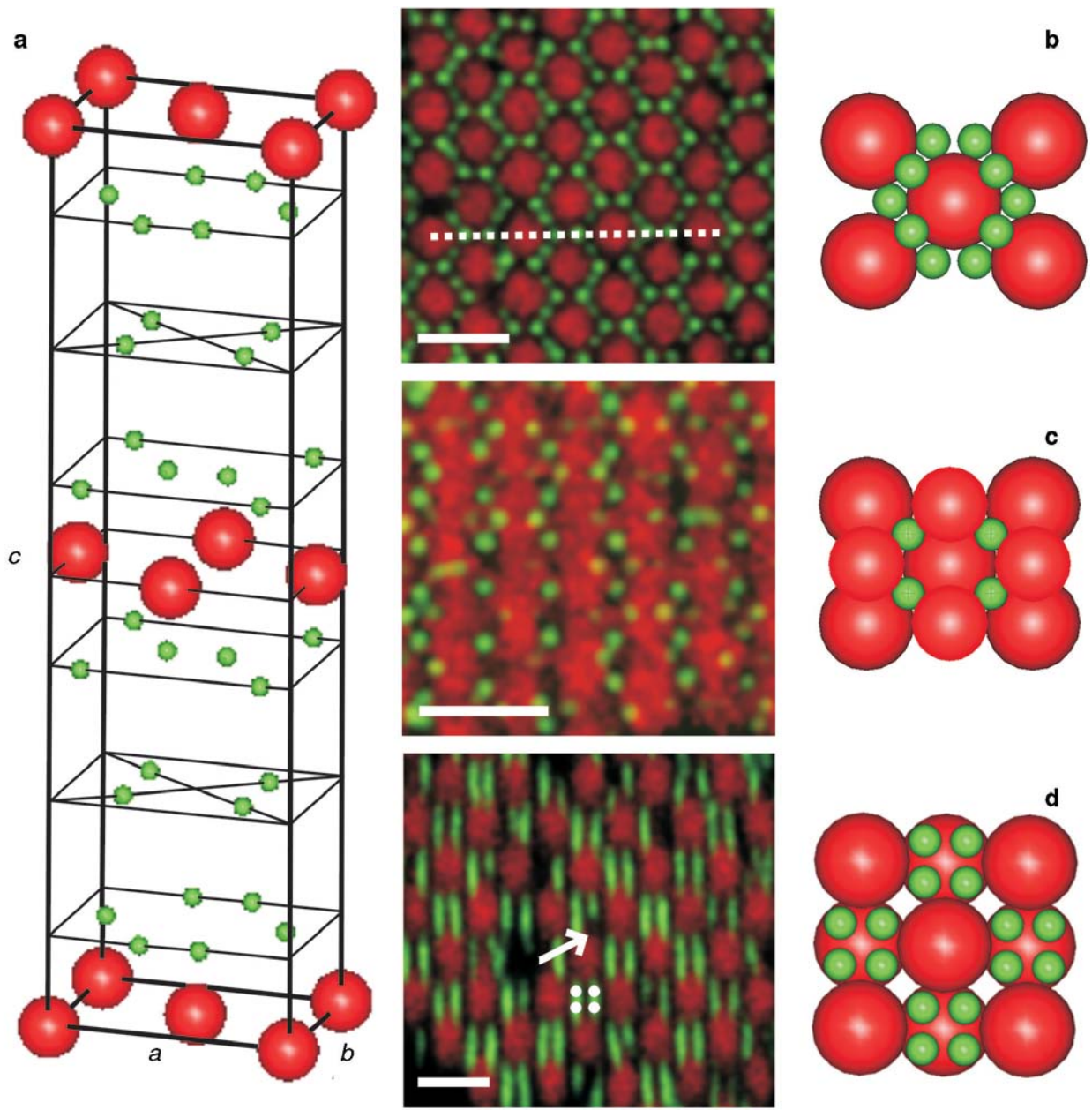

Figure 11. Reprinted with permission from [154]. $\mathrm{AB}_{6}$-type binary crystals. Positive (green) and negative (red) PMMA particles forming a structure with $\mathrm{AB}_{6}$ stoichiometry. (a) Unit cell; (b), (c) confocal images and models showing a layer of large and several layers of small particles (b) and a plane with only small particles (c). (d) Cut along the line in (b). As the microscope could not completely resolve the four small particles in each octahedral hole, their positions are indicated with dots. The arrow indicates a missing particle. All scale bars are $4 \mu \mathrm{m}$.

are chosen well, good control of the stable crystal structure can be achieved. Thus, crystal growth due to heterogeneous nucleation on substrates or due to homogeneous nucleation in an external field is of interest for the production of tailored crystals. Furthermore, tailored substrates can be produced by well-established lithography techniques.

\subsection{Substrates}

Colloidal crystals nucleate much faster on a flat, repulsive wall than in the bulk by homogeneous nucleation. The growth of fcc crystals of hard spheres (HS) on a hard wall has been studied by MC simulation with umbrella sampling, which allows us to measure the nucleation barrier $\Delta G$ [173]. In agreement with experimental observation, the $(1,1,1)$ plane of the fcc crystal is close to completely wetting a hard wall in the simulations. As in the bulk, a larger pressure than at liquidcrystal coexistence is needed for nucleation to start at the wall. However, the nucleation rate is much larger than in the bulk; $\Delta G^{*}$ is found to be two orders of magnitude lower than in the bulk at $p \approx 1.05 p_{\mathrm{c}}$, where $p_{\mathrm{c}}$ is the pressure at fluid-crystal coexistence. The CNT expression for the nucleation barrier can account for the barrier measured in the simulations, if a curvature-dependent line tension $\tau$ is introduced that accounts for the reduced entropy in the region where wall, crystal and fluid meet: $\tau=\tau_{\infty}+c / R . \tau_{\infty}$ is the tension for a straight line and $c / R$ is the curvature correction due to a radius of curvature $R$. $\tau_{\infty}$ is found to be quite close to the rough estimate obtained by assuming that the line tension is due to a surface ring with the height $\sigma$, where $\sigma$ is the spherical diameter. Nucleation on a flat wall is controlled by the line tension. Without the line tension correction, CNT would predict a nucleation barrier $<k_{\mathrm{B}} T$ for volume fractions just above the freezing point $\phi_{\mathrm{f}}=$ 0.494 .

While a flat wall speeds up crystal nucleation of HS, a particle of the same size as the others has no effect on nucleation. Interestingly, simulations suggest that small seed particles with diameters $R \lesssim 5 \sigma$ increase the nucleation barrier, while it is decreased for $R \gtrsim 5 \sigma$ [174]. An increase of $R$ from $5 \sigma$ to $6 \sigma$ reduces $\Delta G$ by almost a factor of three. However, a seed particle of this size is not favorable for growing crystals, since a crystal on a curved surface must contain defects beyond a certain size. Consequently, it is more favorable for the growing crystal to detach from the seed 
particle and the simulations suggest that seed particles of size $5 \sigma<R<6 \sigma$ can act as 'catalysts' for crystal nucleation. As yet, this result has not been verified experimentally. In an experimental study of crystallization close to large spherical impurities no nucleation events were observed $[175,176]$. The final crystal structure was disturbed by the presence of the large particles. The first layer on an impurity was found to be fluid-like and grain boundaries were observed to start at the impurities. For small seed particles it is found that high pressures are needed for nucleation to start. The excess pressure follows a power law: $\left(P-P^{*}\right) \sigma^{3} /\left(k_{\mathrm{B}} T\right)=(\sigma / R)^{0.7}$, where $P^{*}$ is the pressure at freezing on a hard flat wall. These results confirm that particles with $R<5 \sigma$ can be used to suppress heterogeneous crystal nucleation on a wall.

Next to spheres, other relatively simple structures are useful to control the growth of crystals on a surface. Templates of grooves or holes can be used to control the structure and orientation of crystals growing on the surface. This was first done by van Blaaderen et al [177]. A template with small holes arranged as the $(1,0,0)$ fcc plane was used to grow fcc crystals of slowly sedimenting particles without stacking faults. Unlike the $(1,1,1)$ fcc plane, this structure avoids stacking faults, since the positions for the following layers are unambiguous. The effect of grooves can be augmented in systems with depletion interaction, as particles are attracted strongly by the center position of a channel or a cavity [178]. On templates with linear channels, structures with well-defined bond orientational order but low translational order were observed. As in the substrates with cavities forming a square lattice [177], crossing channels allowed us to grow large defect-free fcc crystals.

Straight channels with controlled distance $d$ can be used to grow crystal layers with fcc or bec structure. By varying the volume fraction $\phi$ and adjusting $d$ close to the nearestneighbor distance in the crystal phase $(1.4 \sigma<d<1.5 \sigma)$, the bcc $(1,1,0)$ plane, fcc $(1,0,0)$ plane or the bcc $(1,0,0)$ plane have been obtained on such substrates [179]. One-dimensional order along the channels is obtained for larger distances $d$, when the range of the inter-particle potential is too short for direct interactions of particles in neighboring channels and the indirect interaction via particles in the next layer above is too weak to induce $2 \mathrm{~d}$ order on the template. When $\phi$ is increased, the nearest-neighbor distance decreases and the indirect interaction between particles in adjacent channels can become strong enough to induce crystallization. Interestingly, for $d=2.26 \sigma$ and $\phi=0.24$ a body-centered tetragonal crystal with a height of at least 30 layers is observed. Thus, rather simple templates can be sufficient for growing crystals that are more complex than the ones forming in the bulk. Furthermore, the template causes prefreezing at volume fractions at which the liquid is stable both in the bulk and on a flat wall.

Templates containing small holes that are arranged with the symmetry of an hcp $(1,1,0,0)$ plane have been shown to be an effective tool for growing HS crystals with controlled orientation [180, 181]. An isotropic stretch of the lattice of $\approx 5 \%$ with respect to the equilibrium HS crystal optimizes the order of the crystals. Surprisingly, in the case of an anisotropic stretch by a factor of 1.50 in one direction, a rhombic crystal with non-close-packed structure is observed, which was not predicted for HS crystals.

Analogous templates with a triangular or a rhombic pattern have been studied by simulation [182]. For triangular templates, prefreezing is observed: the first crystal layer forms at a pressure which is about 30\% lower than the pressure for bulk freezing in HS. Freezing of the following layers takes place at monotonically increasing pressures approaching the value for bulk freezing. A rhombic template corresponds to a strained state of the crystal and surface freezing can be suppressed completely, if the strain is above a critical value; this result is expected to be valid for any crystal structure. However, below a threshold strain an otherwise unstable rhombic structure is found to grow with incomplete wetting of the template; this agrees with the experimental observation mentioned above [180,181]. These results are in good agreement with a theory which takes the surface energies, the density difference between crystal and liquid, and the elastic distortion energy due to the template into account. It is predicted that the thickness of the surface crystal grows logarithmically with $\Delta p=p_{\mathrm{c}}-p$ in the case of a triangular template, where $p_{\mathrm{c}}$ is the pressure at crystal-fluid coexistence, and for templates causing a finite strain incomplete crystal surface wetting is found.

Templates consisting of mobile particles can be created with flexibility by optical tweezers, which fix particles to given positions by a harmonic potential [183]. How colloidal particles are trapped in $3 \mathrm{~d}$ and templates are created with tweezers has been demonstrated by Vossen et al [184, 185]. The effectiveness of such templates was studied by MC simulation for templates with structures given by fcc $(1,1,1)$, $(1,1,0)$ and $(1,0,0)$ planes [186]. The template size and positional fluctuations of the particles forming the template were varied analogous to optical traps. The (111) plane is found to be the most effective template and the size of the crystal that forms on the template grows approximately as $N_{\mathrm{T}}^{1 / 3}$, where $N_{\mathrm{T}}$ is the number of particles forming the template; the crystal roughly forms a spherical cap on the template. When the particles forming the template deviate more than $0.05 a$ from their sites, where $a$ is the lattice constant, the effectiveness of the template is reduced and at the Lindemann value, $0.15 a$, the template loses its function as a nucleation site. This suggests that heterogeneous nucleation is not further accelerated by reducing the fluctuations of the template particles to less than 0.05a. A mismatch of the lattice constants of template and bulk crystal is found to reduce the effectiveness of the template; in particular, a smaller lattice spacing of the template leads to a strong reduction of crystal growth. When the CNT expression for $\Delta G$, equation (1), and the effective repulsion between the template-crystal interface and the crystal-fluid interface are taken into account, a crystal with the shape of a spherical cap is expected to form spontaneously on the template. The height of the free energy barrier, $\Delta G^{*}$, for the further nucleation of crystals on this spherical cap is, however, larger than $\Delta G^{*}$ for nucleation on a template covered by fluid, which somewhat reduces the nucleation density rate.

In $2 \mathrm{~d}$ systems, crystals next to a substrate can have longrange order that is supported by the wall, while bulk crystals 
have quasi-long-range order [27]. Furthermore, the nature of the substrate surface also has a strong effect. The effects of two kinds of walls have been studied in a system with an inter-particle potential $\propto r^{-12}$ [187], where $r$ is the distance between particles. When the wall is formed by a crystal of fixed particles, a crystal with long-range order forms on the surface. However, in the case of a smooth wall the structure is fluid-like parallel to the wall and layered in the perpendicular direction; a smectic structure is formed and the long-range order is lost.

\subsection{External fields}

With an applied external field homogeneous nucleation can be controlled throughout the bulk of a system. From the theory side, the treatment of an external potential is an unresolved question, as a difficult non-equilibrium statistical mechanics treatment is required.

In $2 \mathrm{~d}$ systems, the application of an external potential can induce a transition from quasi-long-range to true longrange translational order, if the periodicity of the external field is comparable to that of the crystal [188, 187]. The effect of periodic light fields on the phase behavior has been studied in detail for $2 \mathrm{~d}$ colloidal systems of magnetic particles with repulsive dipole-dipole interaction. In analogy to optical tweezers, two interfering laser beams can create periodic lines in a $2 \mathrm{~d}$ sample that attract colloidal particles. The crystalline region of the phase diagram extends to lower densities, since particles are ordered along the lines of the external potential. In the region that is fluid in the absence of the light field, laser-induced freezing (LIF) is observed [189, 190]. The crystal ordering depends on the interaction between particles in neighboring lines, since this interaction is influenced by the lateral fluctuations of the particles in the direction perpendicular to the field lines. Therefore, the crystal can be re-melted by suppressing the lateral fluctuations of the particles and thereby reducing the interactions between neighboring lines. It was first found from MC computer simulations that such a re-melting can be obtained by applying a stronger external potential that localizes the particles on a narrower line [191]. This laser-induced melting (LIM) effect was confirmed experimentally for crystals formed by LIF [192, 193].

Recently, a binary hard-disc system with size ratio $\alpha=$ 0.414 has been studied by MC simulations with an external field of spatially periodic attractive lines [194, 187]. For a weak external potential, de-mixing of large and small particles in the binary suspension is observed, which leads to the coexistence of ordered structures of large particles and a fluid of small ones. With an increased external potential no de-mixing is found, but a binary $A B$ crystal with a square lattice is found to coexist with a fluid that is also equimolar. Furthermore, the AB crystal is found to be essentially defect-free. This suggests that a suitable external potential can be used to tune the de-mixing of binary hard discs and to grow large defect-free binary crystals.

These studies in $2 \mathrm{~d}$ systems show how the application of an external field can give good control of colloidal suspensions. Even conditions far from equilibrium can be created to study their effect on crystal nucleation. For $3 \mathrm{~d}$ systems, several methods have been developed in recent years.
Large colloidal single crystals can be obtained with a temperature gradient technique [195, 185]. An HS suspension with average volume fraction closely below freezing $(\phi \approx$ 0.493 ) that is placed in a temperature gradient, crystal can be obtained on the cool side, while fluid is stable on the warm side. Large crystals with a diameter $\sim 3 \mathrm{~mm}$ have been grown with $\phi \approx 0.50$ on the cool end of the sample. Due to mechanical equilibrium, the osmotic pressure is constant along the sample, which implies an increase of volume fraction $\phi$ from the warm to the cool end of the sample [196, 197]. This technique could be combined with a structured template as discussed in section 7.1 to obtain large, oriented single crystals [177-181].

Similar control can be achieved by applying a electric field, $E$, that is constant in a well-defined spatial area of a colloidal suspension $[185,198]$. The particles carry induced dipoles that lead to a reduction $-\epsilon_{\mathrm{eff}} E^{2} / 2 V_{\mathrm{p}}$ of their internal energy, if they are located in the region with an electric field. Here, $\epsilon_{\text {eff }}$ is the effective dielectric constant of the particles and $V_{\mathrm{p}}$ is the volume of a particle. With $\epsilon_{\text {eff }}>0$ the particles move towards the region with strong $E$ field due to dielectrophoresis. In HS samples with $\phi \sim 0.50$, crystallization has been induced in the $E$-field region and rhcp crystals have been grown with very sharp crystal-fluid interface. Small $E$ fields are sufficient to obtain a clear density change and, as a consequence, the dipole-dipole interaction between the particles can be neglected in many cases. In a system with negative $\epsilon_{\text {eff }}$, the particles accumulate in the region with $E=0$, which can be an advantage for experimental observation. Far out-ofequilibrium crystallization has been studied in such a system of HS particles [199]. The $E$-field technique is more flexible than the temperature gradient method mentioned above, since it is applicable for many soft matter systems and, furthermore, $\epsilon_{\text {eff }}$ can be controlled by changing the frequency of the applied field. For stronger $E$ fields, the dipole-dipole interaction between the particles can be of the same order of magnitude as other interactions and is not negligible. The particles form strings oriented parallel to the field. As shown in [200], good control can be gained over the structures formed by hard spheres with charge repulsion by controlling both the applied $E$ field and the particle concentration. At relatively low volume fractions, $\phi<0.15$, a fluid of strings was observed to be stable, while at larger $\phi$ more ordered structures are obtained. When the dipole-dipole interaction was dominant along the $E$-field direction but the charge repulsion was stronger in the perpendicular direction, a body-centered tetragonal (bct) structure was formed by the strings of particles and a loosely spaced polycrystalline arrangement of bct crystallites was observed. For a strong $E$ field the dipole-dipole interaction dominates in all directions and a similar bet structure was observed. However, the strings of particles are closer to each other, as was also observed in dipolar hard spheres [201].

Nucleation in the presence of a small laminar shear flow was investigated in Brownian dynamics simulations of charged particles with Yukawa interaction [202, 203]. Since shear can lead to layering promoting crystallization or can remove particles from crystals, the effect of shear on nucleation is not evident. The probability $P(n)$ for finding a nucleus containing $n$ particles was extracted from the simulations and 
$-\log [P(n) / P(1)]$ was calculated. This is not equal to the free energy, because the system is not in equilibrium. An expansion of the chemical potential difference $\Delta \mu$ and surface tension $\gamma$ around equilibrium in terms of the small shear rate $\dot{\gamma}$ was done to analyze the results using equilibrium nucleation theory. It is found that the nucleation barrier increases with $\dot{\gamma}$ and the critical nuclei become larger than in the equilibrium case; small shear is not sufficient to induce layering that boosts crystallization. Under shear the nuclei are found to be predominantly bcc. Their shape is as aspherical as under equilibrium conditions; the longest axis of the nuclei is oriented along the vorticity direction, while the shortest axis lies predominantly along the gradient direction.

A possible application of colloidal crystals that crystallize in an external magnetic field has been explored with charge stabilized magnetite $\left(\mathrm{Fe}_{3} \mathrm{O}_{4}\right)$ nanoparticles [204]. When the magnetic forces are of the same order as the repulsive electrostatic forces, both concentration and phase behavior can be controlled effectively by applying an external magnetic field. The particles are attracted by the region with the highest magnetic field, where their concentration increases and crystals can form; the lattice constant can be controlled by the strength of the magnetic field. Therefore, Bragg reflections of incident light can be tuned; it has been shown that this can be done throughout the visible spectrum. Thus, tunable optical filters have been created.

\section{New directions}

For a deeper understanding of crystal nucleation both in $2 \mathrm{~d}$ and $3 \mathrm{~d}$ the growth process and the structural properties of small crystal nuclei need to be explored in more detail. Direct observations of crystal precursors and critical nuclei crossing the free energy barrier are required. Furthermore, there is a need for characterizations of the core and the interface structure as a function of their size. During recent years it has been shown that direct imaging techniques such as confocal microscopy [74, 73] have great potential for such analyses. The same applies for the investigation of the nucleation in short rods [124]. An improved understanding of the formation of critical nuclei in rods might lead to a general advancement of the knowledge about crystal nucleation. Since crystal nuclei rarely form due to structural fluctuations in an undercooled fluid, methods for controlling their formation should be applied. Optical tweezers are an ideal method for this purpose with colloidal particles [184], since they can be positioned selectively to enhance the formation of a nucleus.

For binary colloidal suspensions the nucleation process is known in even less detail, although the methods for growing tailored binary crystals have been considerably improved in recent years. The goal of growing structures with diamond structure for photonic crystals has not yet been reached, but might be in reach [4].

Microgel particles with soft interactions such as PNIPAM [141] offer opportunities for considerably advancing the knowledge about crystal nucleation. These systems allow us to rapidly change the volume fraction by varying temperature or $\mathrm{pH}$ of the solvent. This allows us to investigate variations of the nucleation process with the depth of a quench from the fluid to the crystal phase. Moreover, such particles are ideal for studying arrested states in order to improve the understanding of the competition between crystallization and the glass transition.

\section{Acknowledgments}

First of all I thank H Löwen for suggesting writing this review article. For the permissions to reproduce figures I thank A van Blaaderen, P Keim, M E Leunissen, Ch Murray and I Snook. I acknowledge financial support from the Adolphe Merkle Foundation.

\section{References}

[1] Pieranski P 1983 Colloidal crystals Contemp. Phys 24 25-73

[2] Dinsmore A D, Crocker J C and Yodh A G 1998 Self-assembly of colloidal crystals Curr. Opin. Colloid Interface Sci. 3 5-11

[3] Soukoulis C M 2001 Photonic Crystals and Light Localization in the 21st Century (NATO Science Series $(C)$ : Mathematical and Physical Sciences) (Dordrecht: Kluwer Academic)

[4] Hynninen A P, Thijssen J H J, Vermolen E C M, Dijkstra M and van Blaaderen A 2007 Self-assembly route for photonic crystals with a bandgap in the visible region Nat. Mater. 6 202-5

[5] Park H S and Xia Y 1999 Assembly of mesoscale particles over large areas and its application in fabricating tunable optical filters Langmuir 15 266-73

[6] Anderson V J and Lekkerkerker H N W 2002 Insights into phase transition kinetics from colloid science Nature 416 811-5

[7] Yethiraj A 2007 Tunable colloids: control of colloidal phase transitions with tunable interactions Nature 3 1099-115

[8] Volmer M and Weber A 1926 Germ-formation in oversaturated figures. Z. Phys. Chem. 119 277-301

[9] Becker R and Doring W 1935 Kinetic treatment of germ formation in supersaturated vapour Ann. Phys. 24 719-52

[10] Turnbull D and Fisher J C 1949 Rate of nucleation in condensed systems J. Chem. Phys. 17 71-3

[11] Kelton K F 1991 Crystal nucleation in liquids and glasses Solid State Physics vol 45, ed H Ehrenbach and D Turnbull (Boston, MA: Academic) pp 75-177

[12] Ostwald W 1897 Studien über die Bildung und Umwandlung fester Körper Z. Phys. Chem. 22289

[13] Kashchiev D 2000 Nucleation: Basic Theory with Applications (Oxford: Butterworth-Heinemann)

[14] Ackerson B J and Schaetzel K 1995 Classical growth of hard-sphere colloidal crystals Phys. Rev. E 52 6448-60

[15] Derber S, Palberg T, Schaetzel K and Vogel J 1997 Growth of a colloidal crystallite of hard spheres Physica A 235204

[16] Lindemann F A 1910 Über die berechnung molekularer eigenfrequenzen Phys. Z. 14 609-12

[17] Strandburg K J 1988 Two-dimensional melting Rev. Mod. Phys. 60 161-207

[18] Zahn K, Lenke R and Maret G 1999 Two-stage melting of paramagnetic colloidal crystals in two dimensions Phys. Rev. Lett. 82 2721-4

[19] Finken R, Schmidt M and Löwen H 2001 Freezing transition of hard hyperspheres Phys. Rev. E $\mathbf{6 5} 016108$

[20] Hansen J-P 1973 Statistical-mechanics of dense ionized matter. 1. Equilibrium properties of classical one-component plasma Phys. Rev. A 8 3096-109

[21] Löwen H, Palberg T and Simon R 1993 Dynamic criterion for freezing of colloidal liquids Phys. Rev. Lett. 70 1557-60 
[22] Truskett T M, Torquato S, Sastry S, Debenedetti P G and Stillinger F H 1998 Structural precursor to freezing in the hard-disk and hard-sphere systems Phys. Rev. E 58 3083-8

[23] Zahn K, Maret G, Russ C and von Grünberg H H 2003 Three-particle correlations in simple liquids Phys. Rev. Lett. 91115502

[24] Kirkwood J E 1951 Phase Transformations in Solids (New York: Wiley)

[25] Peierls R 1935 Quelques proprietes typiques des corps solides Ann. Inst. Henri Poincare 5 177-222

[26] Mermin N D and Wagner H 1966 Absence of ferromagnetism or antiferromagnetism in one- or two-dimensional isotropic Heisenberg models Phys. Rev. Lett. 17 1133-6

[27] Mermin N D 1968 Crystalline order in two dimensions Phys. Rev. 176 250-4

[28] Moucka F and Nezbeda I 2005 Detection and characterization of structural changes in the hard-disk fluid under freezing and melting conditions Phys. Rev. Lett. 94040601

[29] Young A P 1979 Melting and the vector coulomb gas in two dimensions Phys. Rev. B 19 1855-66

[30] Nelson D R and Halperin B I 1979 Dislocation-mediated melting in two dimensions. Phys. Rev. B 19 2457-84

[31] Nelson D R 2002 Defects and Geometry in Condensed Matter Physics (Cambridge: Cambridge University Press)

[32] von Grünberg H-H, Keim P and Maret G 2007 Phase transitions in two-dimensional colloidal systems Soft Matter, Colloidal Order: Entropic and Surface Forces vol 3, ed G Gompper and M Schick (Weinheim: Wiley-VCH) pp 40-83

[33] Reinke D, Stark H, von Grünberg H-H, Schofield A B, Maret G and Gasser U 2007 Noncentral forces in crystals of charged colloids Phys. Rev. Lett. 98038301

[34] Auer S and Frenkel D 2001 Prediction of absolute crystal-nucleation rate in hard-sphere colloids Nature 409 1020-3

[35] Schope H J, Bryant G and van Megen W 2006 Small changes in particle-size distribution dramatically delay and enhance nucleation in hard sphere colloidal suspensions Phys. Rev. E 74060401

[36] Alder B J and Wainwright T E 1957 Phase transition for a hard sphere system J. Chem. Phys. 271208

[37] Wood W W and Jacobson J D 1957 Preliminary results from a recalculation of the Monte Carlo equation of state of hard spheres J. Chem. Phys. 27 1207-8

[38] Frenkel D 1999 Entropy-driven phase transitions Physica A $26326-38$

[39] Hoover W G and Ree F H 1968 Melting transition and communal entropy for hard spheres J. Chem. Phys. 493609

[40] Pusey P N and van Megen W 1986 Phase behaviour of concentrated suspensions of nearly hard colloidal spheres Nature 320 340-2

[41] Cipelletti L and Ramos L 2005 Slow dynamics in glassy soft matter J. Phys.: Condens. Matter 17 R253-85

[42] van Megen W and Underwood S M 1993 Change in crystallization mechanism at the glass transition of colloidal spheres Nature 362 616-8

[43] Zhu J, Li M, Rogers R, Meyer W, Ottenwill R H, STS-73 Space Shuttle Crew, Russel W B and Chaikin P M 1997 Crystallization of hard-sphere colloids in microgravity Nature $387883-5$

[44] Pusey P N 1991 Colloidal suspensions Liquids, Freezing and the Glass Transition, Course 10, Colloidal Suspensions vol 2, ed J P Hansen, D Levesque and J Zinn-Justin (Amsterdam: North-Holland) pp 763-942

[45] Antl L, Goodwin J W, Hill R D, Ottewill R H, Owens S M and Papworth S 1986 The preparation of poly(methyl methacrylate) lattices in non-aqueous media Colloids Surf. 17 67-78
[46] Phan S-E, Russel W B, Cheng Z, Zhu J, Chaikin P M, Dunsmuir J H and Ottewill R H 1996 Phase transition equation of state and limiting shear viscosities of hard sphere dispersions Phys. Rev. E 54 6633-45

[47] Jardine R S and Bartlett P 2002 Synthesis of nonaqueous fluorescent hard-sphere polymer colloids Colloids Surf. 211 127-32

[48] Auer S, Poon W C K and Frenkel D 2003 Phase behavior and crystallization kinetics of poly-12-hydroxystearic-coated polymethylmethacrylate colloids Phys. Rev. E 67020401

[49] Pusey P N, van Megen W, Bartlett P, Ackerson B J, Rarity J G and Underwood S M 1989 Structure of crystals of hard colloidal spheres Phys. Rev. Lett. 63 2753-6

[50] Wilson A J C 1941 Imperfections in the structure of cobalt II. Mathematical treatment of proposed structure Proc. R. Soc. A 180 277-85

[51] Hendricks S and Teller E 1942 X-ray interference in partially ordered layer lattices J. Chem. Phys. 10 147-67

[52] Loose W and Ackerson B J 1994 Model-calculations for the analysis of scattering data from layered structures $J$. Chem. Phys. 101 7211-20

[53] Frenkel D and Ladd A J C 1984 New Monte Carlo method to compute the free energy of arbitrary solids. Application to the fcc and hcp phases of hard spheres J. Chem. Phys. 81 3188-93

[54] Woodcock L V 1997 Entropy difference between the face-centered cubic and hexagonal close-packed crystal structures Nature 385 141-3

[55] Bolhuis P G, Frenkel D, Mau S-C and Huse D A 1997 Entropy difference between crystal phases Nature 388 235-7

[56] Pronk S and Frenkel D 1999 Can stacking faults in hard-sphere crystals anneal out spontaneously? J. Chem. Phys. 110 4589-92

[57] Kegel W K and van Blaaderen A 2000 Direct observation of dynamical heterogeneities in colloidal hard-sphere suspensions Science 287 290-3

[58] Schaetzel K and Ackerson B J 1992 Observation of density fluctuation during crystallization Phys. Rev. Lett. $68337-40$

[59] Schaetzel K and Ackerson B J 1993 Density fluctuations during crystallization of colloids Phys. Rev. E 48 3766-77

[60] He Y, Ackerson B J, van Megen W, Underwood S M and Schaetzel K 1996 Dynamics of crystallization in hard-sphere suspensions Phys. Rev. E 54 5286-97

[61] Dhont J K G, Smits C and Lekkerkerker H N W 1991 A time resolved static light scattering study on nucleation and crystallization in a colloidal system J. Colloid Interface Sci. 152 386-401

[62] Harland J L, Henderson S I, Underwood S M and van Megen W 1995 Observation of accelerated nucleation in dense colloidal fluids of hard sphere particles Phys. Rev. Lett. 75 3572-5

[63] Harland J L and van Megen W 1997 Crystallization kinetics of suspensions of hard colloidal spheres Phys. Rev. E $553054-67$

[64] Cheng Z, Chaikin P M, Zhu J, Russel W B and Meyer W V 2002 Crystallization kinetics of hard spheres in microgravity in the coexistence regime: interactions between growing crystallites Phys. Rev. Lett. 88015501

[65] Schope H J, Bryant G and van Megen W 2006 Two-step crystallization kinetics in colloidal hard-sphere systems Phys. Rev. Lett. 96175701

[66] Wild R and Harrowell P 2001 Density functional theory of the kinetics of crystallization of a hard sphere suspension: coupling structure to density J. Chem. Phys. 114 9059-68

[67] Ackerson B J, Paulin S E, Johnson B, van Megen W and Underwood S 1999 Crystallization by settling in suspensions of hard spheres Phys. Rev. E 59 6903-13

[68] Auer S and Frenkel D 2001 Suppression of crystal nucleation in polydisperse colloids due to increase of the surface free energy Nature 413 711-3 
[69] Shi F G, Tong H Y and Ayers J D 1995 Free energy barrier to nucleation of amorphous-to-crystalline transformation selects the scale of microstructure of crystallized materials Appl. Phys. Lett. 67 350-2

[70] Russel W B, Chaikin P M, Zhu J, Meyer W V and Rogers R 1997 Dendritic growth of hard sphere crystals Langmuir 13 3871-81

[71] Elliot M S, Haddon S B and Poon W C K 2001 Direct observation of pre-critical nuclei in a metastable hard-sphere fluid J. Phys.: Condens. Matter 13 L553-8

[72] Dinsmore A D, Weeks E R, Prasad V, Levitt A C and Weitz D A 2001 Three-dimensional confocal microscopy of colloids Appl. Opt. 40 4152-9

[73] Prasad V, Semwogerere D and Weeks E R 2007 Confocal microscopy of colloids J. Phys.: Condens. Matter 19113102

[74] Gasser U, Weeks E R, Schofield A, Pusey P N and Weitz D A 2001 Real-space imaging of nucleation and growth in colloidal crystallization Science 292 258-62

[75] O'Malley B and Snook I 2003 Crystal nucleation in the hard sphere system Phys. Rev. Lett. 90085702

[76] Yau S-T, Petsev D N, Thomas B R and Vekilov P G 2000 Molecular-level thermodynamic and kinetic parameters for the self-assembly of apoferritin molecules into crystals J. Mol. Biol. 303 667-78

[77] Steinhardt P J, Nelson D R and Ronchetti M 1983 Bond-orientational order in liquids and glasses Phys. Rev. B 28 784-805

[78] Rein ten Wolde P, Ruiz-Montero M J and Frenkel D 1996 Numerical calculation of the rate of crystal nucleation in a Lennard-Jones system at moderate undercooling J. Chem. Phys. 104 9932-47

[79] Gasser U, Wu D T, Schofield A and Weitz D A 2009 Surface tension and structure of crystal nuclei in concentrated colloidal suspensions, in preparation

[80] Marr D W and Gast A P 1993 Planar density-functional approach to the solid-fluid interface of simple liquids Phys. Rev. E 471212

[81] Davidchack R L and Laird B B 2000 Direct calculation of the hard-sphere crystal/melt interfacial free energy Phys. Rev. Lett. 85 4751-4

[82] Ohnesorge R, Löwen H and Wagner H 1994 Density functional theory of crystal-fluid interfaces and surface melting Phys. Rev. E 50 4801-9

[83] Auer S and Frenkel D 2002 Crystallization of weakly charged colloidal spheres: a numerical study J. Phys.: Condens. Matter 14 7667-80

[84] Alexander S and Mctague J 1978 Should all crystals be bcc? Landau theory of solidification and crystal nucleation Phys. Rev. Lett. 41 702-5

[85] Sloutskin E, Lu P J, Kanai T, Schofield A B and Weitz D A 2009 Conformational entropy in crystal nucleation Nature submitted

[86] Hofmeister H 1998 Forty years study of fivefold twinned structures in small particles and thin films Cryst. Res. Technol. 33 3-25

[87] van de Waal B W 1996 Cross-twinning model of fcc crystal growth J. Cryst. Growth 158 153-65

[88] Kofke D A and Bolhuis P G 1999 Freezing of polydisperse hard spheres Phys. Rev. E 59 618-22

[89] Henderson S I and van Megen W 1998 Metastability and crystallization in suspensions of mixtures of hard spheres Phys. Rev. Lett. 80 877-80

[90] Martin S, Bryant G and van Megen W 2005 Crystallization kinetics of polydisperse colloidal hard spheres. II. Binary mixtures Phys. Rev. E 71021404

[91] Martin S, Bryant G and van Megen W 2003 Observation of a smecticlike crystalline structure in polydisperse colloids Phys. Rev. Lett. 90255702

[92] Martin S, Bryant G and van Megen W 2003 Crystallization kinetics of polydisperse colloidal hard spheres: experimental evidence for local fractionation Phys. Rev. E 67061405
[93] Kosterlitz J M and Thouless D J 1973 Ordering, metastability and phase transitions in two-dimensional systems $J$. Phys. C: Solid State Phys. 6 1181-203

[94] Chui S T 1982 Grain-boundary theory of melting in two dimensions Phys. Rev. Lett. 48 933-5

[95] Chui S T 1983 Grain-boundary theory of melting in two dimensions Phys. Rev. B 28 178-94

[96] Kleinert H 1983 Disclinations and first order transitions in 2d melting Phys. Lett. A 95 381-4

[97] Glaser M A and Clark N A 1993 Melting and liquid structure in two dimensions Advances in Chemical Physics vol 83, ed I Prigogine and S A Rice (New York: Wiley) pp 543-709

[98] Chen K, Kaplan T and Mostoller M 1995 Melting in two-dimensional Lennard-Jones systems: observation of a metastable hexatic phase Phys. Rev. Lett. 74 4019-22

[99] Somer F L Jr, Canright G S, Kaplan T, Chen L and Mostoller M 1997 Inherent structures and two-stage melting in two dimensions Phys. Rev. Lett. 79 3431-4

[100] Jaster A 1999 Computer simulation of the two-dimensional melting transition using hard disks Phys. Rev. E 59 2594-602

[101] Mak C H 2006 Large-scale simulations of the two-dimensional melting of hard disks Phys. Rev. E 73065104

[102] Lin S Z, Zheng B and Trimper S 2006 Computer simulations of two-dimensional melting with dipole-dipole interactions Phys. Rev. E 73066106

[103] Murray C A and van Winkle D H 1987 Experimental observation of two-stage melting in a classical two-dimensional screened coulomb system Phys. Rev. Lett. 58 1200-3

[104] Marcus A H and Rice S A 1996 Observations of first-order liquid-to-hexatic and hexatic-to-solid phase transitions in a confined colloid suspension Phys. Rev. Lett. 77 2577-80

[105] Kusner R E, Mann J A, Kerins J and Dahm A J 1994 Two-stage melting of a two-dimensional colloidal lattice with dipole interactions Phys. Rev. Lett. 73 3113-6

[106] Kusner R E, Mann J A and Dahm A J 1995 Two-stage melting in two dimensions in a system with dipole interactions Phys. Rev. B 51 5746-59

[107] Zahn K and Maret G 2000 Dynamic criteria for melting in two dimensions Phys. Rev. Lett. 85 3656-9

[108] von Grünberg H H, Keim P, Zahn K and Maret G 2004 Elastic behavior of a two-dimensional crystal near melting Phys. Rev. Lett. 93255703

[109] Keim P, Maret G and von Grünberg H H 2007 Frank's constant in the hexatic phase Phys. Rev. E 75 031402/1-6

[110] Reis P M, Ingale R A and Shattuck M D 2006 Crystallization of a quasi-two-dimensional granular fluid Phys. Rev. Lett. 96258001

[111] Han Y, Ha N Y, Alsayed A M and Yodh A G 2008 Melting of two-dimensional tunable-diameter colloidal crystals Phys. Rev. E 77041406

[112] Mitus A C, Patashinski A Z, Patrykiejew A and Sokolowski S 2002 Local structure, fluctuations, and freezing in two dimensions Phys. Rev. B 66184202

[113] van Teeffelen S, Likos C N, Hoffmann N and Löwen H 2006 Density functional theory of freezing for soft interactions in two dimensions Europhys. Lett. 75 583-9

[114] van Teeffelen S, Löwen H and Likos C N 2008 Crystallization of magnetic dipolar monolayers: a density functional approach J. Phys.: Condens. Matter 20404217

[115] Eisenmann C, Gasser U, Keim P and Maret G 2005 Pair interaction of dislocations in two-dimensional crystals Phys. Rev. Lett. 95185502

[116] Dillmann P, Maret G and Keim P 2008 Polycrystalline solidification in a quenched $2 \mathrm{~d}$ colloidal system $J$. Phys.: Condens. Matter 20404216

[117] Nagelschmidt G 1938 Rod-shaped clay particles Nature 142114

[118] Dogic Z and Fraden S 2005 Phase behavior of rod-like viruses and virus/sphere mixtures Soft Matter: Complex Colloidal 
Suspensions vol 2, ed G Gompper and M Schick (Weinheim: Wiley-VCH)

[119] Frenkel D and Mulder B M 1985 The hard ellipsoid-of-revolution fluid Mol. Phys. 55 1171-92

[120] Donev A, Stillinger F H, Chaikin P M and Torquato S 2004 Unusually dense crystal packings of ellipsoids Phys. Rev. Lett. 92255506

[121] Pfleiderer P and Schilling T 2007 Simple monoclinic crystal phase in suspensions of hard ellipsoids Phys. Rev. E 75020402

[122] Maeda H and Maeda Y 2003 Liquid crystal formation in suspensions of hard rodlike colloidal particles: direct observation of particle arrangement and self-ordering behavior Phys. Rev. Lett. 90018303

[123] Schilling T and Frenkel D 2004 Self-poisoning of crystal nuclei in hard-rod liquids $J$. Phys.: Condens. Matter 16 S2029-36

[124] Schilling T and Frenkel D 2004 Self-poisoning of crystal nuclei in hard-rod liquids Phys. Rev. Lett. 92085505

[125] Schilling T and Frenkel D 2005 Nucleation in suspensions of anisotropic colloids Comput. Phys. Commun. 169 117-21

[126] Gast A P, Hall C K and Russel W B 1983 Phase separations induced in aqueous colloidal suspensions by dissolved polymer Faraday Discuss. Chem. Soc. 76189

[127] Gast A P, Hall C K and Russel W B 1983 Polymer-induced phase separations in non-aqueous colloidal suspensions J. Colloid Interface Sci. 96 251-67

[128] Gast A P, Russel W B and Hall C K 1986 An experimental and theoretical study of phase transitions in the polystyrene latex and hydroxyethylcellulose system $J$. Colloid Interface Sci. 109 161-71

[129] Lekkerkerker H N W, Poon W C-K, Pusey P N, Stroobants A and Warren P B 1992 Phase behaviour of colloid + polymer mixtures Europhys. Lett. 20 559-64

[130] Hagen M H J and Frenkel D 1994 Determination of phase diagrams for the hard-core attractive Yukawa system J. Chem. Phys. 101 4093-7

[131] Frenkel D, Bladon P, Bolhuis P and Hagen M 1996 Liquid-like behavior in colloidal crystals Physica B 228 33-9

[132] Bolhuis P and Frenkel D 1994 Prediction of an expanded-to-condensed transition in colloidal crystals Phys. Rev. Lett. 72 2211-4

[133] Cacciuto A, Auer S and Frenkel D 2004 Breakdown of classical nucleation theory near isostructural phase transitions Phys. Rev. Lett. 93166105

[134] Mullins W W 1984 Thermodynamic equilibrium of a crystalline sphere in a fluid J. Chem. Phys. 81 1436-42

[135] Cacciuto A and Frenkel D 2005 Stresses inside critical nuclei J. Phys. Chem. B 109 6587-94

[136] Savage J R, Blair D W, Levine A J, Guyer R A and Dinsmore A D 2006 Imaging the sublimation dynamics of colloidal crystallites Science 314 795-8

[137] Debord J D and Lyon L A 2000 Thermoresponsive photonic crystals J. Phys. Chem. B 104 6327-31

[138] Beebe D J, Moore J S, Bauer J M, Yu Q, Liu R H, Devadoss C and Jo B-H 2000 Functional hydrogel structures for autonomous flow control inside microfluidic channels Nature 404 588-90

[139] Vinogradov S V, Bronich T K and Kabanov A V 2002 Nanosized cationic hydrogels for drug delivery: preparation, properties and interactions with cells $A d v$. Drug Deliv. Rev. 54 135-47

[140] Serpe M J, Kim J and Lyon L A 2004 Colloidal hydrogel microlenses Adv. Mater. 16 184-7

[141] Wu J, Zhou B and Hu Z 2003 Phase behavior of thermally responsive microgel colloids Phys. Rev. Lett. 90048304

[142] Watzlawek M, Likos C N and Löwen H 1999 Phase diagram of star polymer solutions Phys. Rev. Lett. 82 5289-92

[143] Gottwald D, Likos C N, Kahl G and Löwen H 2004 Phase behavior of ionic microgels Phys. Rev. Lett. 92068301

[144] Fernandez-Nieves A, de las Nieves F J and Fernandez-Barbero A 2004 Static light scattering from microgel particles: model of variable dielectric permittivity J. Chem. Phys. 120 374-8

[145] Tang S, Hu Z, Cheng Z and Wu J 2004 Crystallization kinetics of thermosensitive colloids probed by transmission spectroscopy Langmuir 20 8858-64

[146] St John A N, Breedveld V and Lyon L A 2007 Phase behavior in highly concentrated assemblies of microgels with soft repulsive interaction potentials J. Phys. Chem. B $1117796-801$

[147] Hellweg T, Dewhurst C D, Brueckner E, Kratz K and Eimer W 2000 Colloidal crystals made of poly(n-isopropylacrylamide) microgel particles Colloid Polym. Sci. 278 972-8

[148] Mohanty P S and Richtering W 2008 Structural ordering and phase behavior of charged microgels J. Phys. Chem. B 112 14692-7

[149] Gasser U, Sierra-Martin B and Fernandez-Nieves A 2009 Crystal structure of highly concentrated ionic microgel suspensions studied by neutron scattering Phys. Rev. E submitted

[150] Sanders J V and Murray M J 1978 Ordered arrangements of spheres of 2 different sizes in opal Nature 275 201-3

[151] Sanders J V 1980 Close-packed structures of spheres of 2 different sizes. 1. Observations on natural opal Phil. Mag. A 42 705-20

[152] Hachisu S and Yoshimura S 1980 Optical demonstration of crystalline superstructures in binary-mixtures of latex globules Nature 283 188-9

[153] Hachisu S and Yoshimura S 1987 Order formation in binary colloids (colloidal alloys in binary latexes) Physics of Complex and Supermolecular Fluids (Exxon Monograph) ed S A Safran and N A Clark (New York: Wiley-Interscience) pp 221-40

[154] Leunissen M E, Christova C G, Hynninen A P, Royall C P, Campbell A I, Imhof A, Dijkstra M, van Roij R and van Blaaderen A 2005 Ionic colloidal crystals of oppositely charged particles Nature $437235-40$

[155] Bartlett P, Ottewill R H and Pusey P N 1990 Freezing of binary mixtures of colloidal hard spheres J. Chem. Phys. 93 1299-312

[156] Bartlett P, Ottewill R H and Pusey P N 1992 Superlattice formation in binary mixtures of hard-sphere colloids Phys. Rev. Lett. 68 3801-4

[157] Mansoori G A, Carnahan N F, Starling K E and Leland T W Jr 1971 Equilibrium thermodynamic properties of the mixture of hard spheres J. Chem. Phys. 54 1523-5

[158] Murray M J and Sanders J V 1980 Close-packed structures of spheres of 2 different sizes. 2. The packing densities of likely arrangements Phil. Mag. A 42 721-40

[159] Eldridge M D, Madden P A and Frenkel D 1993 Entropy-driven formation of a superlattice in a hard-sphere binary mixture Nature $\mathbf{3 6 5} 35-7$

[160] Cottin X and Monson P A 1995 Substitutionally ordered solid-solutions of hard-spheres J. Chem. Phys. 102 3354-60

[161] Trizac E, Eldridge M D and Madden P A 1997 Stability of the ab crystal for asymmetric binary hard sphere mixtures $\mathrm{Mol}$. Phys. 90 675-8

[162] Hunt N, Jardine R and Bartlett P 2000 Superlattice formation in mixtures of hard-sphere colloids Phys. Rev. E 62 900-13

[163] Schofield A B 2001 Binary hard-sphere crystals with the cesium chloride structure Phys. Rev. E 64051403

[164] Christiansion R, Gasser U, Bailey A E, Prasad V, Manley S, Segre P N, Cipelletti L, Weitz D A, Schofield A B, Pusey P N, Doherty M P, Sankaran S, Jankovsky A L, Shiley B, Bowen J, Dendorfer K, Eggers J, Kurta C and Lorik T 2004 Crystallization kinetics of binary alloy colloidal crystals, unpublished

[165] Lant C T, Smart A E, Cannell D S, Meyer W V and Doherty M P 1997 Physics of hard spheres experiment: a general-purpose light-scattering instrument Appl. Opt. 36 7501-7 
[166] Shevchenko E V, Talapin D V, Kotov N A, O'Brien S and Murray C B 2006 Structural diversity in binary nanoparticle superlattices Nature 439 55-9

[167] Bartlett P and Campbell A I 2005 Three-dimensional binary superlattices of oppositely charged colloids Phys. Rev. Lett. 95128302

[168] Bresme F, Vega C and Abascal J L F 2000 Order-disorder transition in the solid phase of a charged hard sphere model Phys. Rev. Lett. 85 3217-20

[169] Hynninen A-P, Christova C G, van Roij R, van Blaaderen A and Dijkstra M 2006 Prediction and observation of crystal structures of oppositely charged colloids Phys. Rev. Lett. 96138308

[170] Underwood S M, van Megen W and Pusey P N 1995 Observation of colloidal crystals with the cesium chloride structure Physica A 221 438-44

[171] Maldovan M, Ullal C K, Carter W C and Thomas E L 2003 Exploring for $3 \mathrm{~d}$ photonic bandgap structures in the $11 \mathrm{fcc}$ space groups Nat. Mater. 2 664-7

[172] Moroz A 2002 Metallo-dielectric diamond and zinc-blende photonic crystals Phys. Rev. B 66115109

[173] Auer S and Frenkel D 2003 Line tension controls wall-induced crystal nucleation in hard-sphere colloids Phys. Rev. Lett. 91015703

[174] Cacciuto A, Auer S and Frenkel D 2004 Onset of heterogeneous crystal nucleation in colloidal suspensions Nature 428 404-6

[175] de Villeneuve V W A, Dullens R P A, Aarts D G A L, Groeneveld E, Scherff J H, Kegel W K and Lekkerkerker H N W 2005 Colloidal hard-sphere crystal growth frustrated by large spherical impurities Science 309 1231-3

[176] de Villeneuve V W A, Verboekend D, Dullens R P A, Aarts D G A L, Kegel W K and Lekkerkerker H N W 2005 Hard sphere crystal nucleation and growth near large spherical impurities J. Phys.: Condens. Matter 17 S3371-8

[177] van Blaaderen A, Ruel R and Wiltzius P 1997 Template-directed colloidal crystallization Nature $385321-4$

[178] Lin Keng-hui, Crocker J C, Prasad V, Schofield A, Weitz D A, Lubensky T C and Yodh A G 2000 Entropically driven colloidal crystallization on patterned surfaces Phys. Rev. Lett. 85 1770-3

[179] Hoogenboom J P, Vossen D L J, Faivre-Moskalenko C, Dogterom M and van Blaaderen A 2002 Patterning surfaces with colloidal particles using optical tweezers Appl. Phys. Lett. $804828-30$

[180] Hoogenboom J P, van Langen-Suurling A K, Romijn J and van Blaaderen A 2003 Hard-sphere crystals with hcp and non-close-packed structure grown by colloidal epitaxy Phys. Rev. Lett. 90138301

[181] Hoogenboom J P, van Langen-Suurling A K, Romijn J and van Blaaderen A 2004 Epitaxial growth of a colloidal hard-sphere hcp crystal and the effects of epitaxial mismatch on crystal structure Phys. Rev. E 69051602

[182] Heni M and Löwen H 2000 Surface freezing on patterned substrates Phys. Rev. Lett. 85 3668-71

[183] Ashkin A 1970 Acceleration and trapping of particles by radiation pressure Phys. Rev. Lett. 24 156-9

[184] Vossen D L J, van der Horst A, Dogterom M and van Blaaderen A 2004 Optical tweezers and confocal microscopy for simultaneous three-dimensional manipulation and imaging in concentrated colloidal dispersions Rev. Sci. Instrum. 75 2960-70

[185] Sullivan M, Zhao K, Harrison C, Austin R H, Megens M, Hollingsworth A, Russel W B, Cheng Z D, Mason T and Chaikin P M 2003 Control of colloids with gravity, temperature gradients, and electric fields $J$. Phys.: Condens. Matter 15 S11-8

[186] Cacciuto A and Frenkel D 2005 Simulation of colloidal crystallization on finite structured templates Phys. Rev. E 72041604

[187] Franzrahe K, Nielaba P, Ricci A, Binder K, Sengupta S, Keim P and Maret G 2008 Field-induced ordering phenomena and non-local elastic compliance in two-dimensional colloidal crystals J. Phys.: Condens. Matter 20404218

[188] Binder K 1995 Phase transitions at surface Cohesion and Structure of Surfaces vol 4, ed F R de Boer and D G Pettifor (Amsterdam: Elsevier) pp 121-283

[189] Chowdhury A and Ackerson B J 1985 Laser-induced freezing Phys. Rev. Lett. 55 833-6

[190] Ackerson B J and Chowdhury A H 1987 Radiation pressure as a technique for manipulating the particle order in colloidal suspensions Faraday Discuss. Chem. Soc. 83 309-17

[191] Chakrabarti J, Krishnamurthy H R, Sood A K and Sengupta S 1995 Reentrant melting in laser field modulated colloidal suspensions Phys. Rev. Lett. 752232

[192] Wei Q-H, Bechinger C, Rudhardt D and Leiderer P 1998 Experimental study of laser-induced melting in two-dimensional colloids Phys. Rev. Lett. 81 2606-9

[193] Bechinger C, Brunner M and Leiderer P 2001 Phase behavior of two-dimensional colloidal systems in the presence of periodic light fields Phys. Rev. Lett. 86930

[194] Franzrahe K and Nielaba P 2007 Entropy versus energy: the phase behavior of a hard-disk mixture in a periodic external potential Phys. Rev. E $76061503-4$

[195] Cheng Z D, Russell W B and Chaikin P M 1999 Controlled growth of hard-sphere colloidal crystals Nature 401 893-5

[196] Carnahan N F and Starling K E 1969 Equation of state for nonattracting rigid spheres J. Chem. Phys. 51 635-6

[197] Hall K R 1972 Another hard-sphere equation of state J. Chem. Phys. 57 2252-4

[198] Sullivan M T, Zhao K, Hollingsworth A D, Austin R H, Russel W B and Chaikin P M 2006 An electric bottle for colloids Phys. Rev. Lett. 96015703

[199] Leunissen M E, Sullivan M T, Chaikin P M and van Blaaderen A 2008 Concentrating colloids with electric field gradients. i. Particle transport and growth mechanism of hard-sphere-like crystals in an electric bottle J. Chem. Phys. 12811

[200] Yethiray A and van Blaaderen A 2003 A colloidal model system with an interaction tunable from hard sphere to soft and dipolar Nature 421 513-7

[201] Dassanayake U, Fraden S and van Blaaderen A 2000 Structure of electrorheological fluids J. Chem. Phys. 112 3851-8

[202] Blaak R, Auer S, Frenkel D and Löwen H 2004 Homogeneous nucleation of colloidal melts under the influence of shearing fields J. Phys.: Condens. Matter 16 S3873-84

[203] Blaak R, Auer S, Frenkel D and Löwen H 2004 Crystal nucleation of colloidal suspensions under shear Phys. Rev. Lett. 93068303

[204] Ge J, Hu Y and Yin Y 2007 Highly tunable superparamagnetic colloidal photonic crystals Angew. Chem. 119 1-5 